\title{
Darboux Integrals for Schrödinger Planar Vector Fields via Darboux Transformations*
}

\author{
Primitivo B. ACOSTA-HUMÁNEZ † and Chara PANTAZI \\ $\dagger$ Departamento de Matemáticas y Estadística Universidad del Norte, \\ Km. 5 via Puerto Colombia, Barranquilla, Colombia \\ E-mail: pacostahumanez@uninorte.edu.co \\ ¥ Departament de Matemàtica Aplicada I, Universitat Politècnica de Catalunya, (EPSEB), \\ Av. Doctor Marañón, 44-50, 08028 Barcelona, Spain \\ E-mail: chara.pantazi@upc.edu
}

Received March 05, 2012, in final form July 06, 2012; Published online July 14, 2012

http://dx.doi.org/10.3842/SIGMA.2012.043

\begin{abstract}
In this paper we study the Darboux transformations of planar vector fields of Schrödinger type. Using the isogaloisian property of Darboux transformation we prove the "invariance" of the objects of the "Darboux theory of integrability". In particular, we also show how the shape invariance property of the potential is important in order to preserve the structure of the transformed vector field. Finally, as illustration of these results, some examples of planar vector fields coming from supersymmetric quantum mechanics are studied.
\end{abstract}

Key words: Darboux theory of integrability; Darboux transformations; differential Galois theory; Schrödinger equation; supersymmetric quantum mechanics

2010 Mathematics Subject Classification: 12H05; 34A30; 34C14; 81Q60; 32S65

\section{Introduction}

We deal with some generalization of the Darboux theory of integrability for planar vector fields and the Darboux transformation of the associated equation.

In 1882, Darboux in his paper [16] presented as a proposition the notable theorem today known as Darboux transformation. This proposition also can be found in his book [17, p. 210]. Curiously Darboux's proposition was forgotten for a long time. In 1926 Ince included it in his book as an exercise (see Exercises 5, 6 and 7 in [22, p. 132]). Ince follows closely Darboux's formulation given in $[16,17]$. P. Dirac, in 1930, published The principles of quantum mechanics, where he gave a mathematically rigorous formulation of quantum mechanics. In 1938, J. Delsarte introduced the notion of transformation (transmutation) operator, today known as intertwining operator and is closely related with Darboux transformation and ladder operators. Later on, in 1941, E. Schrödinger factorized in several ways the hypergeometric equation. It was a byproduct of his factorization method originating an approach that can be traced back to Dirac's raising and lowering operators for the harmonic oscillator. Ten years later, in 1951, another factorization method was presented by L. Infeld and T.E. Hull where they gave the classification of their factorizations of linear second order differential equations for eigenvalue problems of wave mechanics. In 1955, M.M. Crum inspired by Liouville's work about Sturm-Liouville systems and developed one kind of iterative generalization of Darboux transformation. Crum surprisingly did not mention Darboux. In 1971, G.A. Natanzon studied a general form of the transformation that converts the hypergeometric equation to the Schrödinger equation writing down the

\footnotetext{
*This paper is a contribution to the Special Issue "Geometrical Methods in Mathematical Physics". The full collection is available at http://www.emis.de/journals/SIGMA/GMMP2012.html
} 
most general solvable potential, potential for which the Schrödinger equation can be reduced to hypergeometric or confluent hypergeometric form, concept introduced by himself.

Almost one hundred years after Darboux's proposition, in 1981, Edward Witten with his renowned paper [45] gave birth to the supersymmetric quantum mechanics and he discussed general conditions for dynamical supersymmetry breaking. Since Witten's work we can found in the literature a big amount of papers related to supersymmetric quantum mechanics. Maybe the most relevant of these papers was written in 1983 by L.É. Gendenshtein, where he presented the shape invariance condition, i.e. the preservation of the shape under Darboux transformation. Gendenshtein used this property to find the complete spectra for a broad class of problems including all known exactly solvable problems of quantum mechanics (bound state and reflectionless potentials). Today this kind of exactly solvable potentials satisfying the shape invariance condition are called shape invariant potentials, see [20]. In 2009, in [1,3] were presented a Galoisian point of view of the supersymmetric quantum mechanics and in particular of the Darboux transformations and shape invariance condition. We point out that the first analysis of Darboux transformations from the differential Galois point of view was done by V.P. Spiridonov in [40]. The present work follows these approaches with the same point of view.

From the other hand, Darboux in 1878 presented a simple way to construct first integrals and integrating factors for planar polynomial vector fields, see [15]. The key point of his method are the invariant algebraic curves of such vector fields. His approach has been related with problems concering limit cycles, centers and bifurcation problems, see for instance [21, 31, 38]. Moreover, the geometric scenario of the algebraic curves determines the structure of the vector fields, see [10, 11, 35]. Nowdays Darboux's method has been improved for polynomial vector fields basically taking into account the multiplicity of the invariant algebraic curves see for instance $[9,11,13]$. Moreover, the existence of a rational first integral and Darboux's method are related by Jouanolou's results, see [23, 32]. Prelle and Singer [36, 39] gave the relation between elementary/Liouvillian first integrals and integrating factors that are constructed by Darboux's method. Additionally, Darboux's ideas have been extended to a particular class of non-autonomous vector fields, see [5, 30].

Our main aim in this paper is to relate Darboux's theory of integrability for planar vector fields of Riccati type with Schrödinger equation and Darboux transformation of the associated equation. So we consider polynomial vector fields of the form

$$
\dot{v}=d v / d t=S_{0}(x)+S_{1}(x) v+S_{2}(x) v^{2}, \quad \dot{x}=d x / d t=N(x),
$$

with $S_{0}, S_{1}, S_{2} \in \mathbb{C}[x]$. In particular for non-relativistic quantum mechanics we have $S_{2}(x)=$ $-N(x), S_{1}(x)=0$ and $S_{0}(x)=N(x)(V(x)-\lambda)$ with $V(x)=T(x) / N(x), N, T \in \mathbb{C}[x]$ and $\lambda$ a constant.

Hence, we deal with systems of the form

$$
\dot{v}=d v / d t=N(x)\left(V(x)-\lambda-v^{2}\right), \quad \dot{x}=d x / d t=N(x),
$$

or equivalently we can consider the polynomial vector field

$$
\dot{v}=d v / d t=T(x)-N(x) \lambda-N(x) v^{2}, \quad \dot{x}=d x / d t=N(x),
$$

of degree $m=\max \{\operatorname{deg} T(x), \operatorname{deg} N(x)+2\}$. Note that the associated foliation of system (1) is

$$
v^{\prime}=\frac{d v}{d x}=V(x)-\lambda-v^{2},
$$

with $V \in \mathbb{C}(x)$, see also [2].

The structure of the paper is the following: In Section 2 we present the basic concepts of differential Galois theory, Schrödinger equation, Darboux transformation and Darboux's theory 
of integrability of planar polynomial vector fields. In Section 3 we present our main results. More concrete, in Proposition 3 we characterize the differential Galois group of the Schrödinger equation with the class of the first integral of the corresponding vector field. Additionally, in Proposition 4 we present a condition for the non-existence of a rational first integral. In Proposition 5 we show how we can construct the generalized Darboux first integrals and integrating factors of the transformed vector field using a solution of the initial Schrödinger equation. In Theorem 9 we show that the strong isogaloisian property of the Darboux transformation and the shape invariance property of the potential are necessary in order to preserve the rational structure of the elements of the transformed vector field. At the end, in Section 4 we give several examples as applications of our results.

As far as we know this is the first time that is presented in the literature the link between Darboux theory of integrability of planar vector fields and Darboux transformation of the associated Schrödinger equation.

\section{Theoretical background}

In this section we present the theoretical background that we use in this work. Most of the results are naturally extended in higher dimension.

\subsection{Differential Galois theory}

We start regarding an algebraic model for functions and the corresponding Galois theory known as differential Galois theory or also Picard-Vessiot theory, see [26, 27, 37, 43, 44] for all detail. The following preliminaries correspond to a quick overview of this theory and can be found also in $[1,3]$.

Definition 1 (differential fields). Let $F$ be a commutative field of characteristic zero. A derivation of $F$ is a map $\frac{d}{d x}: F \rightarrow F$ satisfying

$$
\frac{d}{d x}(a+b)=\frac{d a}{d x}+\frac{d b}{d x}, \quad \frac{d}{d x}(a \cdot b)=\frac{d a}{d x} \cdot b+a \cdot \frac{d b}{d x},
$$

for all $a, b \in F$. We say that $\left(F, \frac{d}{d x}\right)$ (or just $F$ when there is no ambiguity) is a differential field with the derivation $\frac{d}{d x}$.

We assume that $F$ contains an element $x$ such that $\frac{d}{d x}(x)=1$. Let $\mathcal{C}$ be the field of constants of $F: \mathcal{C}=\left\{c \in F \mid \frac{d c}{d x}=0\right\}$. $\mathcal{C}$ is of characteristic zero and will be assumed to be algebraically closed.

Throughout this paper, the coefficient field for a differential equation will be defined as the smallest differential field containing all the coefficients of the equation.

In particular we deal with second order linear homogeneous differential equations, i.e., equations of the form

$$
\frac{d^{2} y}{d x^{2}}+\alpha \frac{d y}{d x}+\beta y=0, \quad \alpha, \beta \in F .
$$

Definition 2 (Picard-Vessiot extension). Consider the differential equation (4). Let $L$ be a differential field containing $F$ (a differential extension of $F$ ). We say that $L$ is a PicardVessiot extension of $F$ for the differential equation (4) if there exist two linearly independent solutions of the differential equation (4) namely $y_{1}, y_{2} \in L$ such that $L=F\left\langle y_{1}, y_{2}\right\rangle$ (i.e. $L=$ $\left.F\left(y_{1}, y_{2}, d y_{1} / d x, d y_{2} / d x\right)\right)$ and moreover $L$ and $F$ have the same field of constants $\mathcal{C}$. 
In what follows, we work with Picard-Vessiot extensions and the term "solution of (4)" will mean "solution of (4) in $L$ ". So any solution of the differential equation (4) is a linear combination (over $\mathcal{C}$ ) of $y_{1}$ and $y_{2}$.

Definition 3 (differential Galois groups). An $F$-automorphism $\sigma$ of the Picard-Vessiot extension $L$ is called a differential automorphism if

$$
\sigma\left(\frac{d a}{d x}\right)=\frac{d}{d x}(\sigma(a)) \quad \forall a \in L \quad \text { and } \quad \sigma(a)=a \quad \forall a \in F .
$$

The group of all differential automorphisms of $L$ over $F$ is called the differential Galois group of $L$ over $F$ and is denoted by $\operatorname{DGal}(L / F)$.

Given $\sigma \in \operatorname{DGal}(L / F)$, we see that $\left\{\sigma y_{1}, \sigma y_{2}\right\}$ are also solutions of the equation (4). Hence there exists a matrix $A_{\sigma} \in \mathrm{GL}(2, \mathbb{C})$, such that

$$
\sigma\left(\left(\begin{array}{ll}
y_{1} & y_{2}
\end{array}\right)\right)=\left(\begin{array}{ll}
\sigma\left(y_{1}\right) & \sigma\left(y_{2}\right)
\end{array}\right)=\left(\begin{array}{ll}
y_{1} & y_{2}
\end{array}\right) A_{\sigma} .
$$

As $\sigma$ commutes with the derivation, this extends naturally to an action on a fundamental solution matrix of the companion first order system associated with the equation (4). We have

$$
\sigma\left(\left(\begin{array}{cc}
y_{1} & y_{2} \\
\frac{d y_{1}}{d x} & \frac{d y_{2}}{d x}
\end{array}\right)\right)=\left(\begin{array}{cc}
\sigma\left(y_{1}\right) & \sigma\left(y_{2}\right) \\
\sigma\left(\frac{d y_{1}}{d x}\right) & \sigma\left(\frac{d y_{2}}{d x}\right)
\end{array}\right)=\left(\begin{array}{cc}
y_{1} & y_{2} \\
\frac{d y_{1}}{d x} & \frac{d y_{2}}{d x}
\end{array}\right) A_{\sigma} .
$$

This defines a faithful representation $\operatorname{DGal}(L / K) \rightarrow \mathrm{GL}(2, \mathbb{C})$ and it is possible to consider $\operatorname{DGal}(L / K)$ as a subgroup of $\mathrm{GL}(2, \mathbb{C})$ and depends on the choice of the fundamental system $\left\{y_{1}, y_{2}\right\}$ only up to conjugacy.

Recall that an algebraic group $G$ is an algebraic manifold endowed with a group structure. Let $\operatorname{GL}(2, \mathbb{C})$ denote, as usual, the set of invertible $2 \times 2$ matrices with entries in $\mathbb{C}$ (and $\operatorname{SL}(2, \mathbb{C})$ be the set of matrices with determinant equal to 1 ). A linear algebraic group will be a subgroup of $\mathrm{GL}(2, \mathbb{C})$ equipped with a structure of algebraic group. One of the fundamental results of the Picard-Vessiot theory is the following theorem (see $[26,27])$.

Theorem 1. The differential Galois group $\mathrm{DGal}(L / F)$ is an algebraic subgroup of $\mathrm{GL}(2, \mathbb{C})$.

In fact, the differential Galois group measures the algebraic relations between the solutions (and their derivatives) of the differential equation (4). It is sometimes viewed as the object which should tell "what algebra sees of the dynamics of the solutions".

In an algebraic group $G$, the largest connected algebraic subgroup of $G$ containing the identity, noted $G^{\circ}$, is a normal subgroup of finite index. It is often called the connected component of the identity. If $G=G^{0}$ then $G$ is a connected group.

When $G^{0}$ satisfies some property, we say that $G$ virtually satisfies this property. For example, virtually solvability of $G$ means solvability of $G^{0}$ (see [44]).

Theorem 2 (Lie-Kolchin). Let $G \subseteq \mathrm{GL}(2, \mathbb{C})$ be a virtually solvable group. Then $G^{0}$ is triangularizable, i.e. it is conjugate to a subgroup of upper triangular matrices.

Throughout this work we will use the following definition.

Definition 4 (Liouvillian integrability). We say that the linear differential equation (4) is (Liouville) integrable if the Picard-Vessiot extension $L \supset F$ is obtained as a tower of differential fields $F=L_{0} \subset L_{1} \subset \cdots \subset L_{m}=L$ such that $L_{i}=L_{i-1}(\eta)$ for $i=1, \ldots, m$, where either

1) $\eta$ is algebraic over $L_{i-1}$, that is $\eta$ satisfies a polynomial equation with coefficients in $L_{i-1}$; 
2) $\eta$ is primitive over $L_{i-1}$, that is $\frac{d \eta}{d x} \in L_{i-1}$;

3) $\eta$ is exponential over $L_{i-1}$, that is $\left(\frac{d \eta}{d x}\right) / \eta \in L_{i-1}$.

We remark that in the usual terminology of differential algebra for integrable equations the corresponding Picard-Vessiot extensions are called Liouvillian. From now on we say that an equation is integrable whether it is integrable in the sense of differential Galois theory according to Definition 4. The following theorem is due to Kolchin.

Theorem 3. The equation (4) is integrable if and only if $\mathrm{DGal}(L / F)$ is virtually solvable.

There is an algorithm due to Kovacic [28] that decides about the integrability of the equation (4) in the case where $F=\mathbb{C}(x)$. In practice, Kovacic's algorithm deals with the reduced form of equation (4), namely with the form $y^{\prime \prime}=r y$, where $r$ is a rational function. Kovacic used the fact that $\operatorname{DGal}(L / F) \subseteq \mathrm{SL}(2, \mathbb{C})$, in order to separate his algorithm in three cases for the integrability of the equation (4):

Case 1. $\mathrm{DGal}(L / F)$ is reducible,

Case 2. $\operatorname{DGal}(L / F)$ is irreducible,

Case 3. $\operatorname{DGal}(L / F)$ is finite primitive.

\subsection{The Schrödinger equation}

Here we first introduce the Schrödinger equation and then we present the preliminaries about Schrödinger equation from a Galoisian point of view, see [1,3].

In classical mechanics for a particle of mass $m$ moving under the action of a potential $U$ the Hamiltonian is given by

$$
H=\frac{\|\vec{p}\|^{2}}{2 m}+U(\vec{x}), \quad \vec{p}=\left(p_{1}, \ldots, p_{n}\right), \quad \vec{x}=\left(x_{1}, \ldots, x_{n}\right),
$$

and corresponds to the energy (kinetic plus potential). From the other hand in quantum mechanics the momentum $\vec{p}$ is given by $\vec{p}=-\imath \hbar \nabla$, where $\hbar$ is the Planck constant and $\nabla$ is the Laplacian operator. In this case the Hamiltonian operator $H$ is the Schrödinger (non-relativistic, stationary) operator which is given by

$$
H=-\frac{\hbar^{2}}{2 m} \nabla^{2}+V(\vec{x})
$$

where $\vec{x}$ is the coordinate and $V(\vec{x})$ is the potential or potential energy. The Schrödinger equation is given by $H \Psi=\lambda \Psi$, where the eigenfunction $\Psi$ is the wave function and the eigenvalue $\lambda \in \operatorname{Spec}(H)$ is the energy level. The solutions $\Psi$ of the Schrödinger equation are the states of the particle and $\operatorname{Spec}(H)$ denotes the spectrum of the operator $H$. In [41] it can be found the details about the mathematical foundations of quantum mechanics for the Schrödinger equation.

According to $[14,45]$, a supersymmetric quantum mechanical system is one in which there are operators $Q_{i}$ that commute with the Hamiltonian $\mathcal{H}$ and satisfying

$$
\begin{aligned}
& {\left[Q_{i}, \mathcal{H}\right]=Q_{i} H-H Q_{i}=0,} \\
& \left\{Q_{i}, Q_{j}\right\}=Q_{i} Q_{j}+Q_{j} Q_{i}=\delta_{i j} \mathcal{H} \quad \text { and } \quad \delta_{i j}= \begin{cases}1, & i=j, \\
0, & i \neq j .\end{cases}
\end{aligned}
$$


For $n=2$, we obtain the simplest example of a supersymmetric quantum mechanical system. In this case we have that $x \in \mathbb{R}$. Thus, the supercharges $Q_{i}$ are defined as

$$
Q_{ \pm}=\frac{\sigma_{1} p \pm \sigma_{2} W(x)}{2}, \quad Q_{+}=Q_{1}, \quad Q_{-}=Q_{2}
$$

where $p=-i \hbar \frac{d}{d x}, W: \mathbb{R} \longrightarrow \mathbb{R}$ is the superpotential and $\sigma_{i}$ are the Pauli spin matrices.

The operator $\mathcal{H}$, satisfying $Q_{i} \mathcal{H}=\mathcal{H} Q_{i}$ and $2 Q_{i}^{2}=\mathcal{H}$, is given by

$$
\mathcal{H}=\frac{I_{2} p^{2}+I_{2} W^{2}(x)+\hbar \sigma_{3} \frac{d}{d x} W(x)}{2}=\left(\begin{array}{cc}
H_{+} & 0 \\
0 & H_{-}
\end{array}\right), \quad I_{2}=\left(\begin{array}{ll}
1 & 0 \\
0 & 1
\end{array}\right) .
$$

The operators $H_{-}$and $H_{+}$are the supersymmetric partner Hamiltonians and are given by

$$
H_{ \pm}=-\frac{1}{2} \frac{d^{2}}{d x^{2}}+V_{ \pm}, \quad V_{ \pm}=\left(\frac{W}{\sqrt{2}}\right)^{2} \pm \frac{1}{\sqrt{2}} \frac{d}{d x}\left(\frac{W}{\sqrt{2}}\right)
$$

where $V_{ \pm}$are the supersymmetric partner potentials.

Now we follow $[1,3]$ in order to present the Schrödinger equation in the context of differential Galois theory. Thus, the Schrödinger equation (stationary and one dimensional) now is written as

$$
H \Psi=\lambda \Psi, \quad H=-\frac{d^{2}}{d x^{2}}+V(x), \quad V \in F,
$$

where $F$ is a differential field (with $\mathcal{C}=\mathbb{C}$ as field of constants). We will deal with the integrability of equation (6) in agreement with our definition of integrability, i.e., in the sense of differential Galois theory, see Definition 4. In [1,3] were introduced the following notations, useful for our purposes.

- $\Lambda \subseteq \mathbb{C}$ denotes the algebraic spectrum of $H$, i.e., the set of eigenvalues $\lambda$ such that equation (6) is integrable (Definition 4).

- $L_{\lambda}$ denotes the Picard-Vessiot extension of equation (6). Thus, the differential Galois group of (6) is denoted by $\operatorname{DGal}\left(L_{\lambda} / K\right)$.

Definition 5 (algebraically solvable and quasi-solvable potentials). The potential $V(x) \in F$ is:

- an algebraically solvable potential when $\Lambda$ is an infinite set, or

- an algebraically quasi-solvable potential when $\Lambda$ is a non-empty finite set, or

- an algebraically non-solvable potential when $\Lambda=\varnothing$.

When $\operatorname{Card}(\Lambda)=1$, we say that $V(x) \in F$ is a trivial algebraically quasi-solvable potential.

The following theorem shows that if there exist more than one eigenvalue in the algebraic spectrum of the Schrödinger operator with $F=\mathbb{C}(x)$, then we cannot fall in case 3 of Kovacic's algorithm.

Theorem 4 (see $[1,3])$. Consider the Schrödinger equation (6) with $F=\mathbb{C}(x)$ and PicardVessiot extension $L_{\lambda}$. If $\mathrm{DGal}\left(L_{0} / F\right)$ is finite primitive, then $\mathrm{DGal}\left(L_{\lambda} / F\right)$ is not finite primitive for all $\lambda \in \Lambda \backslash\{0\}$.

From [1,3] note that the known cases of rational potentials in quantum mechanics leads to Schrödinger equations falling in case 1 of Kovacic's algorithm. Additionally, if Card $(\Lambda)>1$ then any algebraic solution of the Riccati equation associated to the Schrödinger equation (6) is a root of a polynomial of degree at most two. 


\subsection{Darboux transformation}

Darboux gave in [16] a transformation that allow us to transform some type of differential equations into other differential equations preserving the type. The following results corresponds to the Darboux transformation, denoted as DT, in the Galoisian and quantum mechanic formalism, see $[1,3]$.

Theorem 5 (Galoisian version of DT). Assume $H_{ \pm}=-\frac{d^{2}}{d x^{2}}+V_{ \pm}(x)$ and $\Lambda \neq \varnothing$. Consider the Schrödinger equation $H_{-} \Psi^{(-)}=\lambda \Psi^{(-)}$with $V_{-}(x) \in F$. Let DT be the transformation such that $V_{-} \mapsto V_{+}, \Psi^{(-)} \mapsto \Psi^{(+)}, F \mapsto \tilde{F}$. Then for the Schrödinger equation $H_{+} \Psi^{(+)}=\lambda \Psi^{(+)}$with $V_{+}(x) \in \widetilde{F}$ the following statements holds:

i) $\mathrm{DT}\left(V_{-}\right)=V_{+}=\Psi_{\lambda_{1}}^{(-)} \frac{d^{2}}{d x^{2}}\left(\frac{1}{\Psi_{\lambda_{1}}^{(-)}}\right)+\lambda_{1}=V_{-}-2 \frac{d^{2}}{d x^{2}}\left(\ln \Psi_{\lambda_{1}}^{(-)}\right)$,

$$
\operatorname{DT}\left(\Psi_{\lambda_{1}}^{(-)}\right)=\Psi_{\lambda_{1}}^{(+)}=\frac{1}{\Psi_{\lambda_{1}}^{(-)}} \text {, where }
$$

$\Psi_{\lambda_{1}}^{(-)}$is a particular solution of $H_{-} \Psi^{(-)}=\lambda_{1} \Psi^{(-)}, \lambda_{1} \in \Lambda$;

ii) $\operatorname{DT}\left(\Psi_{\lambda}^{(-)}\right)=\Psi_{\lambda}^{(+)}=\frac{d}{d x} \Psi_{\lambda}^{(-)}-\frac{d}{d x}\left(\ln \Psi_{\lambda_{1}}^{(-)}\right) \Psi_{\lambda}^{(-)}, \lambda \neq \lambda_{1}$, where

$\Psi_{\lambda}^{(-)}$is the general solution of $H_{-} \Psi^{(-)}=\lambda \Psi^{(-)}$for $\lambda \in \Lambda \backslash\left\{\lambda_{1}\right\}$ and

$\Psi_{\lambda}^{(+)}$is the general solution of $H_{+} \Psi^{(+)}=\lambda \Psi^{(+)}$also for $\lambda \in \Lambda \backslash\left\{\lambda_{1}\right\}$.

Remark 1. According to $[1,3]$ we have that a transformation is called isogaloisian whether it preserves the differential Galois group: the initial equation and the transformed equation have the same differential Galois group. Furthermore, when the differential field and the PicardVessiot extension are preserved, then the transformation is called strong isogaloisian.

In agreement with Theorem 5 and Remark 1, we obtain the following results, see [1, 3] for complete statements and proofs.

Proposition 1. In general, DT is isogaloisian and virtually strong isogaloisian. Furthermore, if $\partial_{x}\left(\ln \Psi_{\lambda_{1}}^{(-)}\right) \in F$, then DT is strong isogaloisian.

Proposition 2. The supersymmetric partner potentials $V_{ \pm}$are rational functions if and only if the superpotential $W$ is a rational function.

Corollary 1. The superpotential $W \in \mathbb{C}(x)$ if and only if DT is strong isogaloisian.

Remark 2. The examples of this paper are in agreement with the previous results, since $\widetilde{F}=F$ due to the fact that the superpotential $W$ belongs to $F=\mathbb{C}(x)$. The following definition is a partial Galoisian adaptation of the original definition given in [20] $(F=\mathbb{C}(x))$. The complete Galoisian adaptation is given when $F$ is any differential field, see [1,3].

Definition 6 (rational shape invariant potentials, see $[1,3]$ ). Assume $V_{ \pm}(x ; \mu) \in \mathbb{C}(x ; \mu)$, where $\mu$ is a family of parameters. The potential $V=V_{-} \in \mathbb{C}(x)$ is said to be rational shape invariant potential with respect to $\mu$ and $\lambda=\lambda_{n}$ being $n \in \mathbb{Z}_{+}$, if there exists a function $f$ such that

$$
V_{+}\left(x ; a_{0}\right)=V_{-}\left(x ; a_{1}\right)+R\left(a_{1}\right), \quad a_{1}=f\left(a_{0}\right), \quad \lambda_{n}=\sum_{k=2}^{n+1} R\left(a_{k}\right), \quad \lambda_{0}=0 .
$$

Hence the form of the potentials $V_{ \pm}$are preserved up to parameters. Theorem 5 and Propositions 1 and 2 lead us to following result. 
Theorem 6 (see $[1,3]$ ). Consider $H \Psi^{(-)}=\lambda_{n} \Psi^{(-)}$with Picard-Vessiot extension $L_{n}$, where $n \in \mathbb{Z}_{+}$. If $V=V_{-} \in \mathbb{C}(x)$ is a shape invariant potential with respect to $\lambda=\lambda_{n}$, then

$$
\operatorname{DGal}\left(L_{n+1} / \mathbb{C}(x)\right)=\operatorname{DGal}\left(L_{n} / \mathbb{C}(x)\right), \quad n>0 .
$$

Hence for rational shape invariant potentials Galois group is preserved due to Picard-Vessiot extension and differential field.

\subsection{Darboux's theory of integrability for planar polynomial vector fields}

In this subsection we present the basic ideas of Darboux's method for planar polynomial vector fields, see [15]. We don't give en extensive presentation of this theory but we only present the basic results that we need in Section 3.

We consider the polynomial (differential) system in $\mathbb{C}^{2}$ defined by

$$
\frac{d x}{d t}=\dot{x}=P(x, y), \quad \frac{d y}{d t}=\dot{y}=Q(x, y),
$$

where $P$ and $Q$ are polynomials in the variables $x$ and $y$. The independent variable $t$ can be real or complex. We associate to the polynomial differential system (7) the polynomial vector field

$$
X=P(x, y) \frac{\partial}{\partial x}+Q(x, y) \frac{\partial}{\partial y}
$$

and its associated foliation is given by $P d y-Q d x=0$.

An algebraic curve $f(x, y)=0$ in $\mathbb{C}^{2}$ with $f \in \mathbb{C}[x, y]$ is an invariant algebraic curve of the vector field (8) if

$$
X(\log (f))=K
$$

for some polynomial $K \in \mathbb{C}[x, y]$ called the cofactor of the invariant algebraic curve $f=0$. Note that due to the definition (9) we have that the degree of the cofactor $K$ is less than the degree of the polynomial vector field (7). Moreover, the curve $f=0$ is formed by trajectories of the vector field $X$.

For a given system (7) of degree $m$ the computation of all the invariant algebraic curves is a very hard problem because in general we don't know about the maximum degree of such curves. However, imposing additionally conditions either for the structure of the system or for the nature of the curves we can have an evidence of a such a bound $[7,8,12]$.

Let $h, g \in \mathbb{C}[x, y]$ be relatively prime in the ring $\mathbb{C}[x, y]$. The function $\exp (g / h)$ is called an exponential factor of the polynomial system (7) if there is a polynomial $L \in \mathbb{C}[x, y]$ (also called cofactor) that satisfies the equation $X(g / h)=L$. It turns out that if $h$ is not a constant polynomial, then $h=0$ is one of the invariant algebraic curve of (7).

The following theorem is a short version of Darboux theory of integrability for planar polynomial differential systems. For more details and also for higher dimension see [29]. For generalizations see [5].

Theorem 7. Suppose that a polynomial system (7) of degree $m$ admits

- $p$ irreducible invariant algebraic curves $f_{i}=0$ with cofactors $K_{i}$ for $i=1, \ldots, p$,

- $q$ exponential factors $F_{j}$ with cofactors $L_{j}$ for $j=1, \ldots, q$.

Then the following statements hold: 
(a) The function

$$
f_{1}^{\lambda_{1}} \cdots f_{p}^{\lambda_{p}} F_{1}^{\mu_{1}} \cdots F_{q}^{\mu_{q}}
$$

is a first integral of (7) if and only if $\sum_{i=1}^{p} \lambda_{i} K_{i}+\sum_{i=1}^{q} \mu_{i} L_{i}=0$.

(b) The function (10) is a (Darboux) integrating factor of the vector field (8) if and only if $\sum_{i=1}^{p} \lambda_{i} K_{i}+\sum_{i=1}^{q} \mu_{i} L_{i}+\left(P_{x}+Q_{y}\right)=0$.

Integrating factors for planar systems can be thought as parametrization of the independent variable (time) that yields to a divergence free system.

As we show in Theorem 7 for polynomial vector fields the invariant algebraic curves and (because of their multiplicity, see [13]) the exponential factors are the basic elements in order to construct first integrals/integrating factors. As we will see the method of Theorem 7 also works for more general expressions of vector fields, curves, exponential factors and cofactors, see also $[5,18,19]$.

Definition 7 (generalized exponential factor). Consider $S(x) \in \mathbb{C}(x)$. We define a generalized exponential factor of a polynomial vector field $X$ any expression of the form $F=\exp \left(\int S(x)\right)$ which satisfies $X(F)=L F$ and $L$ is called generalized cofactor.

Definition 8 (generalized Darboux function). We define generalized Darboux function any expression of the form

$$
\begin{aligned}
& \left(y-S_{1}(x)\right)^{\lambda_{1}} \cdots\left(y-S_{p}(x)\right)^{\lambda_{p}} \exp \left(\int S(x)\right) \\
& \quad=\left(y-S_{1}(x)\right)^{\lambda_{1}} \cdots\left(y-S_{p}(x)\right)^{\lambda_{p}} \exp (\tilde{S}(x)) \prod\left(x-x_{i}\right)^{b_{i}},
\end{aligned}
$$

with $S_{i}, S, \tilde{S}, g \in \mathbb{C}(x), x_{i}, b_{i}, \lambda_{i} \in \mathbb{C}$ and $p \in \mathbb{Z}_{+}$, see also [46].

An integrating factor of the form (11) will be called generalized Darboux integrating factor and similarly a first integral of the form (11) will be called generalized Darboux first integral.

Remark 3. We consider the Schrödinger equation (6) with $F=\mathbb{C}(x)$ and the associated Ricatti equation (3). We also consider $v_{1}(x), v_{2}(x), v_{3}(x)$ particular solutions of equation (3) with $V \in \mathbb{C}(x)$ and we write $V(x)=T(x) / N(x)$ with $T, N \in \mathbb{C}[x]$. Note that an associated polynomial vector field to equation (3) can be written into the form (2).

(a) Following $[2,28,46]$ we can distinguish the following cases about the type of the first integrals of equation (3) or equivalently of the polynomial vector field (2).

Case 1. ( $i$ ) If only $v_{1}(x) \in \mathbb{C}(x)$ then the vector field (2) has a first integral of DarbouxSchwarz-Christoffel type, namely a first integral of the form

$$
I(v, x)=\frac{1}{y-v_{1}(x)} \exp (g(x)) \prod\left(x-x_{i}\right)^{a_{i}}+\int^{x} \exp (g(u)) \prod\left(u-x_{i}\right)^{a_{i}-m_{i}} P(u) d u,
$$

with $P \in \mathbb{C}[x], g \in \mathbb{C}(x), x_{i}, a_{i} \in \mathbb{C}$ and $m_{i} \in \mathbb{Z}_{+}$, see also [46].

(ii) If both $v_{1}(x), v_{2}(x) \in \mathbb{C}(x)$ then the vector field (2) has a generalized Darboux first integral of the form

$$
I(v, x)=\frac{-v+v_{2}(x)}{-v+v_{1}(x)} \exp \left(\int\left(v_{2}(x)-v_{1}(x)\right) d x\right) .
$$


Furthermore if the differential Galois group of the Schrödinger equation (6) is a cyclic group of order $k$ then the $k$-th power of the first integral (12) is rational, see also [2].

Case 2. If $v_{1}$ is a solution of a quadratic polynomial then the vector field (2) has a first integral of hyperelliptic type.

Case 3. If all $v_{1}, v_{2}, v_{3}$ are algebraic over $\mathbb{C}(x)$ then the vector field (2) has a rational first integral of the form

$$
I(v, x)=\frac{\left(v_{2}-v_{1}\right)\left(v_{1}-v\right)}{\left(v_{3}-v_{1}\right)\left(v_{2}-v\right)}
$$

(b) In general, knowing one algebraic solution $v_{1}(x)$ of equation (3) we can obtain a second solution of (3), namely

$$
v_{2}(x)=v_{1}+\frac{\exp \left(-2 \int v_{1} d x\right)}{\int \exp \left(-2 \int v_{1} d x\right) d x}
$$

Then, the vector field (2) has always a first integral of the form (12) and can be rewritten either as Darboux-Schwarz-Christoffel type or as a generalized Darboux function or as a first integral of hyperelliptic type or as a rational first integral.

Additionally in [2] appears the following result about the existence of a rational first integral of a polynomial vector field whose foliation is of Riccati type.

Theorem 8. The Galois group of the equation $y^{\prime \prime}=r(x) y$ with $r \in \mathbb{C}(x)$ is finite if and only if there exist a rational first integral for the associated polynomial vector field of the corresponding Riccati equation.

Note that the Schrödinger equation (6) can be always written into the form $y^{\prime \prime}=r(x) y$ and so we can always apply Theorem 8 .

\section{Main results}

From now on we consider the Schrödinger equation (6) with potential $V=V_{-}$:

$$
\begin{array}{ll}
\Psi^{(-) \prime \prime}=\left(V_{-}(x)-\lambda\right) \Psi^{(-)}, & V_{-}=\frac{T}{N}, \\
\lambda \in \Lambda, \quad \operatorname{Card}(\Lambda)>1, & F=\mathbb{C}(x) .
\end{array}
$$

After the change of coordinates $\zeta^{(-)}=\Psi^{(-) \prime} / \Psi^{(-)}$, equation (6) can be written as

$$
\zeta^{(-) \prime}=V_{-}-\lambda-\zeta^{(-) 2}
$$

and we associate to equation (14) the polynomial vector field

$$
X_{\lambda}^{(-)}=\left(T-\lambda N-N \zeta^{(-) 2}\right) \frac{\partial}{\partial \zeta^{(-)}}+N \frac{\partial}{\partial x}
$$

of degree $m=\max \{\operatorname{deg} T(x), \operatorname{deg} N(x)+2\}$. By Propositions 1, 2 and Corollary 1, we have

that for given $V_{-} \in F$ and $W=\zeta_{0}^{(-)}=\Psi_{0}^{(-) \prime} / \Psi_{0}^{(-)} \in F$ then we obtain that $V_{+} \in F$, i.e., the Darboux transformation DT is strong isogaloisian. Moreover, the superpotential $W$ is rational. The applications considered in this work correspond to this case.

The following proposition follows directly by Remark 3 and [2]. 
Proposition 3. The differential Galois group $\operatorname{DGal}\left(L_{\lambda} / F\right)$ of the Schrödinger equation (13) is virtually solvable if and only if the first integral of the vector field (15) can be written in one of the forms appearing in Remark 3(a).

In the next proposition we present a result about the non-existence of a rational first integral.

Proposition 4. Consider the Schrödinger equation (13). If $\mathrm{DGal}\left(L_{\lambda} / F\right)$ is not cyclic then the associated foliation of the Schrödinger equation (13) has not rational first integrals.

Proof. It follows directly from Theorems 4 and 8 .

Lemma 1. Consider $\Psi_{\lambda}^{(-)}(x)$ a solution of the Schrödinger equation (13) and we denote by

$$
\zeta_{\lambda}^{(-)}(x)=\frac{\left(\Psi_{\lambda}^{(-)}(x)\right)^{\prime}}{\Psi_{\lambda}^{(-)}(x)}=\left(\ln \left(\Psi_{\lambda}^{(-)}(x)\right)\right)^{\prime}, \quad \lambda \in \Lambda .
$$

Then for all $\lambda \in \Lambda$ the vector field (15) admits the following:

- Invariant curve

$$
f_{\lambda}^{(-)}\left(\zeta^{(-)}, x\right)=-\zeta^{(-)}+\zeta_{\lambda}^{(-)}(x)
$$

with generalized cofactor

$$
K_{\lambda}^{(-)}\left(\zeta^{(-)}, x\right)=-N(x)\left(\zeta^{(-)}+\zeta_{\lambda}^{(-)}(x)\right)
$$

- Generalized exponential factor

$$
F_{\lambda}^{(-)}\left(\zeta^{(-)}, x\right)=\exp \left(\int\left(\frac{1}{2} \frac{N^{\prime}(x)}{N(x)}+\zeta_{\lambda}^{(-)}(x)\right) d x\right)
$$

with generalized cofactor

$$
L_{\lambda}^{(-)}\left(\zeta^{(-)}, x\right)=N^{\prime}(x) / 2+N(x) \zeta_{\lambda}^{(-)}(x) .
$$

- An integrating factor of the form

$$
R_{\lambda}^{(-)}\left(\zeta^{(-)}, x\right)=\frac{\exp \left(\int\left(-\frac{N^{\prime}(x)}{N(x)}-2 \zeta_{\lambda}^{(-)}(x)\right) d x\right)}{\left(-\zeta^{(-)}+\zeta_{\lambda}^{(-)}(x)\right)^{2}} .
$$

- A first integral of the form

$$
I_{\lambda}^{(-)}\left(\zeta^{(-)}, x\right)=\frac{-\zeta^{(-)}+\zeta_{(\lambda, 2)}^{(-)}}{-\zeta^{(-)}+\zeta_{(\lambda, 1)}^{(-)}} \exp \left(\int\left(\zeta_{(\lambda, 2)}^{(-)}-\zeta_{(\lambda, 1)}^{(-)}\right) d x\right)
$$

with $\zeta_{(\lambda, 1)}$ as in (16) and

$$
\zeta_{(\lambda, 2)}^{(-)}=\zeta_{(\lambda, 1)}^{(-)}+\frac{\exp \left(-2 \int \zeta_{(\lambda, 1)}^{(-)} d x\right)}{\int \exp \left(-2 \int \zeta_{(\lambda, 1)}^{(-)} d x\right) d x} .
$$


Proof. Note that $\zeta_{\lambda}^{(-)}(x)$ is a solution of the associated equation $\zeta^{(-)^{\prime}}=V_{-}(x)-\lambda-\zeta^{(-)^{2}}$ for all $\lambda \in \Lambda$. Using the expression of the vector field (15) we have

$$
\begin{aligned}
X_{\lambda}^{(-)}\left(f_{\lambda}^{(-)}\left(\zeta^{(-)}, x\right)\right) & =\left(T-\lambda N-N \zeta^{(-) 2}\right)(-1)+N \zeta_{\lambda}^{(-) \prime} \\
& =N\left(\zeta^{(-) 2}-\zeta_{\lambda}^{(-) 2}\right)=K_{\lambda}^{(-)}\left(\zeta^{(-)}, x\right) \cdot f_{\lambda}^{(-)}\left(\zeta^{(-)}, x\right) .
\end{aligned}
$$

Note that the curve $f_{\lambda}^{(-)}$is polynomial in the variable $\zeta^{(-)}$but it could be not polynomial in the variable $x$. Direct computations shows that system (14) admits the generalized exponential factor

$$
F_{\lambda}^{(-)}\left(\zeta^{(-)}, x\right)=\exp \left(\int\left(\frac{1}{2} \frac{N^{\prime}(x)}{N(x)}+\zeta_{\lambda}^{(-)}(x)\right) d x\right)
$$

with generalized cofactor $L_{\lambda}^{(-)}\left(\zeta^{(-)}, x\right)=N^{\prime}(x) / 2+\zeta_{\lambda}^{(-)}(x)$. Note that vector field (15) has divergence $\operatorname{div}\left(X_{\lambda}\right)=-2 N \zeta^{(-)}+N^{\prime}(x)$. Hence, we have that $-2 K_{\lambda}^{(-)}-2 L_{\lambda}^{(-)}+\operatorname{div}\left(X_{\lambda}^{(-)}\right)=0$ and similarly to Theorem $7(b)$ we have that $R_{\lambda}^{(-)}=\left(f_{\lambda}^{(-)} F_{\lambda}^{(-)}\right)^{-2}$ is an integrating factor for the vector field (15).

If $\Psi_{(\lambda, 1)}^{(-)}(x)$ a solution of the Schrödinger equation $(13)$ then $\zeta_{(\lambda, 1)}^{(-)}$is a solution of the Riccati equation (14). Then, according to Remark $(3)(b)$ we have that $\zeta_{(\lambda, 2)}^{(-)}$is another solution of the Riccati equation (14). In this case the vector field (15) admits the two invariant curves

$$
f_{(\lambda, 1)}^{(-)}\left(\zeta^{(-)}, x\right)=-\zeta^{(-)}+\zeta_{(\lambda, 1)}^{(-)}(x), \quad f_{(\lambda, 2)}^{(-)}\left(\zeta^{(-)}, x\right)=-\zeta^{(-)}+\zeta_{(\lambda, 2)}^{(-)}(x),
$$

with generalized cofactors

$$
K_{(\lambda, 1)}^{(-)}\left(\zeta^{(-)}, x\right)=-N(x)\left(\zeta^{(-)}+\zeta_{(\lambda, 1)}^{(-)}(x)\right), \quad K_{(\lambda, 2)}^{(-)}\left(\zeta^{(-)}, x\right)=-N(x)\left(\zeta^{(-)}+\zeta_{(\lambda, 2)}^{(-)}(x)\right) .
$$

The two generalized exponential factors

$$
\begin{aligned}
& F_{(\lambda, 1)}^{(-)}\left(\zeta^{(-)}, x\right)=\exp \left(\int\left(\frac{1}{2} \frac{N^{\prime}(x)}{N(x)}+\zeta_{\lambda, 1}^{(-)}(x)\right) d x\right), \\
& F_{(\lambda, 2)}^{(-)}\left(\zeta^{(-)}, x\right)=\exp \left(\int\left(\frac{1}{2} \frac{N^{\prime}(x)}{N(x)}+\zeta_{\lambda, 2}^{(-)}(x)\right) d x\right) .
\end{aligned}
$$

with generalized cofactors

$$
L_{(\lambda, 1)}^{(-)}\left(\zeta^{(-)}, x\right)=N^{\prime}(x) / 2+N(x) \zeta_{\lambda, 1}^{(-)}(x), \quad L_{(\lambda, 2)}^{(-)}\left(\zeta^{(-)}, x\right)=N^{\prime}(x) / 2+N(x) \zeta_{\lambda, 2}^{(-)}(x) .
$$

Note that it holds $-K_{(\lambda, 1)}^{(-)}+K_{(\lambda, 2)}^{(-)}-L_{(\lambda, 1)}^{(-)}+L_{(\lambda, 2)}^{(-)}=0$, and similar to Theorem $7(a)$ we have that the vector field $X_{\lambda}^{(-)}$admits the first integral

$$
I_{(\lambda)}^{(-)}=\frac{f_{(\lambda, 2)}^{(-)} \cdot F_{(\lambda, 2)}^{(-)}}{f_{(\lambda, 1)}^{(-)} \cdot F_{(\lambda, 1)}^{(-)}} .
$$

Hence, the lemma is proved.

Proposition 5. Consider $\Psi_{0}^{(-)}(x)$ a particular solution of the Schrödinger equation $H_{-} \Psi^{(-)}=$ $\lambda \Psi^{(-)}$for $\lambda=0$ and let $\zeta_{0}^{(-)}(x)=\left(\ln \left(\Psi_{0}^{(-)}(x)\right)\right)^{\prime}$. Then after the Darboux transformation DT for all $\lambda \neq 0$ equation (14) becomes

$$
\zeta^{(+) \prime}=V_{+}-\lambda-\zeta^{(+) 2}
$$


with $V_{+}=-\zeta_{0}{ }^{(-) \prime}+\zeta_{0}{ }^{(-) 2}$ and its associated vector field is

$$
X_{\lambda}^{(+)}=\left(-\zeta_{0}^{(-) \prime}+\zeta_{0}^{(-) 2}-\lambda-\zeta^{(+) 2}\right) \frac{\partial}{\partial \zeta^{(+)}}+\frac{\partial}{\partial x} .
$$

The vector field (18) for all $\lambda \neq 0$ admits the following:

- Invariant curve

$$
f_{\lambda}^{(+)}\left(\zeta^{(+)}, x\right)=-\zeta^{(+)}+\zeta_{\lambda}^{(-)}+\left(\ln \left(\zeta_{\lambda}^{(-)}-\zeta_{0}^{(-)}\right)\right)^{\prime},
$$

with generalized cofactor

$$
K_{\lambda}^{(+)}\left(\zeta^{(+)}, x\right)=-\zeta^{(+)}-\zeta_{\lambda}^{(-)}-\left(\ln \left(\zeta_{\lambda}^{(-)}-\zeta_{0}^{(-)}\right)\right)^{\prime}
$$

- Generalized exponential factor

$$
F_{\lambda}^{(+)}\left(\zeta^{(+)}, x\right)=\left(\zeta_{\lambda}^{(-)}-\zeta_{0}^{(-)}\right) \exp \left(\int \zeta_{\lambda}^{(-)}\right)
$$

with generalized cofactor

$$
L_{\lambda}^{(+)}=\zeta_{\lambda}^{(-)}+\left(\ln \left(\zeta_{\lambda}^{(-)}-\zeta_{0}^{(-)}\right)\right)^{\prime}
$$

- An integrating factor $($ for $\lambda \neq 0)$

$$
R_{\lambda}^{(+)}\left(\zeta^{(+)}, x\right)=\frac{\exp \left(-2 \int \zeta_{\lambda}^{(-)}\right)}{\left(\zeta_{\lambda}^{(-)}-\zeta_{0}^{(-)}\right)^{2}\left(-\zeta^{(+)}+\zeta_{\lambda}^{(-)}+\left(\ln \left(\zeta_{\lambda}^{(-)}-\zeta_{0}^{(-)}\right)\right)^{\prime}\right)^{2}}
$$

- A first integral of the form

$$
\begin{aligned}
I_{\lambda}^{(+)}\left(\zeta^{(+)}, x\right)= & \frac{-\zeta^{(+)}+\zeta_{(\lambda, 2)}^{(-)}+\left(\ln \left(\zeta_{(\lambda, 2)}^{(-)}-\zeta_{0}^{(-)}\right)\right)^{\prime}}{-\zeta^{(+)}+\zeta_{(\lambda, 1)}^{(-)}+\left(\ln \left(\zeta_{(\lambda, 1)}^{(-)}-\zeta_{0}^{(-)}\right)\right)^{\prime}} \\
& \times\left(\frac{\zeta_{(\lambda, 2)}^{(-)}-\zeta_{0}^{(-)}}{\zeta_{(\lambda, 1)}^{(-)}-\zeta_{0}^{(-)}}\right) \exp \left(\int\left(\zeta_{(\lambda, 2)}^{(-)}-\zeta_{(\lambda, 1)}^{(-)}\right) d x\right) .
\end{aligned}
$$

Proof. For $\lambda=0$ we consider $\Psi_{0}^{(-)}$a particular solution of the Schrödinger equation $H_{-} \Psi^{(-)}=$ $\lambda \Psi^{(-)}$. We denote by $\zeta_{0}^{(-)}=\left(\ln \left(\Psi_{0}^{-}\right)\right)^{\prime}$. Then from Theorem 5 for $\lambda \neq 0$ we have

$$
\Psi_{\lambda}^{(+)}=\Psi_{\lambda}^{(-) \prime}-\zeta_{0}^{(-)} \Psi_{\lambda}^{(-)}=\exp \left(\int \zeta_{\lambda}^{(-)}\right)\left(\zeta_{\lambda}^{(-)}-\zeta_{0}^{(-)}\right)
$$

and we have use that $\zeta_{\lambda}^{(-)}=\Psi_{\lambda}^{(-)^{\prime}} / \Psi_{\lambda}^{(-)}$. Note that $\Psi_{\lambda}^{(+)}$is a solution of the Schrödinger equation $H_{+} \Psi^{(+)}=\lambda \Psi^{(+)}$. Let

$$
\zeta_{\lambda}^{(+)}=\left(\ln \left(\Psi_{\lambda}^{(+)}\right)\right)^{\prime}=\left(\ln \left(\exp \left(\int \zeta_{\lambda}^{(-)}\right)\left(\zeta_{\lambda}^{(-)}-\zeta_{0}^{(-)}\right)\right)\right)^{\prime} .
$$

According to Theorem 5 we have

$$
V_{+}=\Psi_{0}^{(-)}\left(\frac{1}{\Psi_{0}^{(-)}}\right)^{\prime \prime}=\Psi_{0}^{(-)}\left(-\frac{\zeta_{0}^{(-)}}{\Psi_{0}^{(-)}}\right)^{\prime}=-\zeta_{0}^{(-) \prime}+\zeta_{0}^{(-) 2},
$$


and we additionally consider the change of variables $\zeta^{(+)}=\left(\ln \left(\Psi^{(+)}\right)\right)^{\prime}$. Then the Schrödinger equation $H_{+} \Psi^{(+)}=\lambda \Psi^{(+)}$falls in equation (17).

Therefore, similar to Lemma 1 , the vector field (18) for all $\lambda \neq 0$ admits the invariant curve $f_{\lambda}^{(+)}$with generalized cofactor $K_{\lambda}^{(+)}$given by

$$
\begin{aligned}
& f_{\lambda}^{(+)}\left(\zeta^{(+)}, x\right)=-\zeta^{(+)}+\zeta_{\lambda}^{(+)}=-\zeta^{(+)}+\zeta_{\lambda}^{(-)}+\left(\ln \left(\zeta_{\lambda}^{(-)}-\zeta_{0}^{(-)}\right)\right)^{\prime}, \\
& K_{\lambda}^{(+)}\left(\zeta^{(+)}, x\right)=-\zeta^{(+)}-\zeta_{\lambda}^{(+)}=-\zeta^{(+)}-\zeta_{\lambda}^{(-)}-\left(\ln \left(\zeta_{\lambda}^{(-)}-\zeta_{0}^{(-)}\right)\right)^{\prime} .
\end{aligned}
$$

Additionally, for all $\lambda \neq 0$ admits the generalized exponential factor

$$
\begin{aligned}
F_{\lambda}^{(+)}\left(\zeta^{(+)}, x\right) & =\exp \left(\int \zeta_{\lambda}^{(+)}\right)=\exp \left(\int\left(\zeta_{\lambda}^{(-)}+\left(\ln \left(\zeta_{\lambda}^{(-)}-\zeta_{0}^{(-)}\right)\right)^{\prime}\right)\right) \\
& =\left(\zeta_{\lambda}^{(-)}-\zeta_{0}^{(-)}\right) \exp \left(\int \zeta_{\lambda}^{(-)}\right) .
\end{aligned}
$$

with generalized cofactor $L_{\lambda}^{(+)}=\zeta_{\lambda}^{(+)}=\zeta_{\lambda}^{(-)}+\left(\ln \left(\zeta_{\lambda}^{(-)}-\zeta_{0}^{(-)}\right)\right)^{\prime}$. Note that the vector field (18) has divergence $\operatorname{div} X=-2 \zeta^{(+)}$and for all $\lambda \neq 0$ it holds $-2 K_{\lambda}^{(+)}-2 L_{\lambda}^{(+)}+\operatorname{div} X_{\lambda}{ }^{(+)}=0$, and similarly to Theorem $7(b)$ we have that $R_{\lambda}^{(+)}=f_{\lambda}^{(+)} F_{\lambda}{ }^{(+)^{-2}}$.

Note that if $\Psi_{(\lambda, 1)}^{(+)}$is a solution of the Schrödinger equation $H_{+} \Psi^{(+)}=\lambda \Psi^{(+)}$then $\zeta_{(\lambda, 1)}^{(+)}$is a solution of the Riccati equation $\zeta_{\lambda}^{(+) \prime}=V_{+}-\lambda-\zeta^{(+) 2}$. Following the same arguments as in the proof of Lemma 1 we obtain the expression of the first integral. Hence, the proof is completed.

Theorem 9. If Darboux transformation is strong isogaloisian for a potential $V_{-} \in \mathbb{C}(x)$, then each pair of functions $f_{\lambda}^{( \pm)}\left(\zeta^{( \pm)}, x\right), K_{\lambda}^{( \pm)}\left(\zeta^{( \pm)}, x\right), L_{\lambda}^{( \pm)}\left(\zeta^{( \pm)}, x\right)$ and $I_{\lambda}^{( \pm)}\left(\zeta^{( \pm)}, x\right)$ belong to the differential extension in the variables $\zeta^{( \pm)}$and $x$. Furthermore, if $V_{-}$is a rational shape invariant potential, then $f_{\lambda}^{( \pm)}\left(\zeta^{( \pm)}, x\right), K_{\lambda}^{( \pm)}\left(\zeta^{( \pm)}, x\right)$ and $L_{\lambda}^{( \pm)}\left(\zeta^{( \pm)}, x\right)$ are rational functions in $\zeta^{( \pm)}$and $x$.

Proof. The proof follows directly by Theorem 6 and Proposition 5 .

\section{Applications in quantum mechanics}

In this section we present some examples that relate polynomial vector fields, according to Lemma 1 and Proposition 5, with supersymmetric quantum mechanics. Schrödinger equations with potentials such as free particle (explicitly discussed in [40] with a similar approach), three dimensional harmonic oscillator and Coulomb potential are analyzed as Schrödinger vector fields.

Example 1 (free particle). Consider the Schrödinger equation $H_{-} \Psi^{(-)}=\lambda \Psi^{(-)}$with potential $V_{-}=0$ and differential field ${ }^{1} F=\mathbb{C}(x)$. Thus, we have that $\Lambda=\mathbb{C}$.

If we choose $\lambda_{0}=0$ and as particular solution of the Schrödinger equation the solution $\Psi_{0}^{(-)}=x$ we have that $\zeta_{0}^{(-)}=\Psi_{0}^{(-)^{\prime}} / \Psi_{0}^{(-)}=1 / x$. Since $\zeta_{0}^{(-)} \in \mathbb{C}(x)$ from Proposition 1 we have that $D T$ is strong isogaloisian. Additionally,

$$
\mathrm{DT}\left(V_{-}\right)=V_{+}=-\zeta_{0}^{(-) \prime}+\zeta_{0}^{(-) 2}=\frac{2}{x^{2}},
$$

and so $V_{-}$is not shape invariant. Then for $\lambda \neq 0$ the general solution of $H_{-} \Psi^{(-)}=\lambda \Psi^{(-)}$is given by

$$
\Psi_{\lambda}^{(-)}=c_{1} \Psi_{(\lambda, 1)}^{(-)}+c_{2} \Psi_{(\lambda, 2)}^{(-)},
$$

\footnotetext{
${ }^{1}$ Although the smallest differential field containing the coefficients of the Schrödinger equation is $\mathbb{C}$, to avoid triviality, we choose $\mathbb{C}(x)$ as the suitable differential field for such equation.
} 
where we have consider $\Psi_{(\lambda, 1)}^{(-)}=\exp (\sqrt{-\lambda} x)$ and $\Psi_{(\lambda, 2)}^{(-)}=\exp (-\sqrt{-\lambda} x)$. Hence we have that

$$
\begin{aligned}
& \zeta_{\lambda}^{(-)}=\left(\ln \left(\Psi_{\lambda}^{(-)}\right)\right)^{\prime}=\sqrt{-\lambda} \frac{c_{1} \exp (\sqrt{-\lambda} x)-c_{2} \exp (-\sqrt{-\lambda} x)}{c_{1} \exp (\sqrt{-\lambda} x)+c_{2} \exp (-\sqrt{-\lambda} x)}, \\
& \zeta_{0}^{(-)}=\left(\ln \left(\Psi_{0}^{(-)}\right)\right)^{\prime}=\frac{1}{x} .
\end{aligned}
$$

Additionally, we have that

$$
\zeta_{(\lambda, 1)}^{(-)}=\left(\ln \left(\Psi_{(\lambda, 1)}^{(-)}\right)\right)^{\prime}=\sqrt{-\lambda} \quad \text { and } \quad \zeta_{(\lambda, 2)}^{(-)}=\left(\ln \left(\Psi_{(\lambda, 2)}^{(-)}\right)\right)^{\prime}=-\sqrt{-\lambda} .
$$

Here in the expression of the vector field (15) we have that $T(x)=0$ and $N(x)=1$. Hence the polynomial vector field (15) associated to the Schrödinger equation $H_{-} \Psi^{(-)}=\lambda \Psi^{(-)}$with potential $V_{-}=0$, is

$$
X_{\lambda}^{-}=\left(-\lambda-\zeta^{(-) 2}\right) \frac{\partial}{\partial \zeta^{(-)}}+\frac{\partial}{\partial x},
$$

and is quadratic. Then according to Lemma 1 for all $\lambda \in \Lambda \backslash\{0\}$, the vector field $X_{\lambda}^{(-)}$admits the following:

Invariant curve

$$
f_{\lambda}^{(-)}\left(\zeta^{(-)}, x\right)=-\zeta^{(-)}+\sqrt{-\lambda} \frac{c_{1} \exp (\sqrt{-\lambda} x)-c_{2} \exp (-\sqrt{-\lambda} x)}{c_{1} \exp (\sqrt{-\lambda} x)+c_{2} \exp (-\sqrt{-\lambda} x)},
$$

and $f_{\lambda}^{(-)}\left(\zeta^{(-)}, x\right) \in \mathbb{C}\left(\zeta^{(-)}, x, \exp (\sqrt{-\lambda} x)\right)$ with generalized cofactor

$$
K_{\lambda}^{(-)}\left(\zeta^{(-)}, x\right)=-\zeta^{(-)}-\sqrt{-\lambda} \frac{c_{1} \exp (\sqrt{-\lambda} x)-c_{2} \exp (-\sqrt{-\lambda} x)}{c_{1} \exp (\sqrt{-\lambda} x)+c_{2} \exp (-\sqrt{-\lambda} x)}
$$

and $K_{\lambda}^{(-)}\left(\zeta^{(-)}, x\right) \in \mathbb{C}\left(\zeta^{(-)}, x, \exp (\sqrt{-\lambda} x)\right)$.

Generalized exponential factor

$$
F_{\lambda}^{(-)}\left(\zeta^{(-)}, x\right)=c_{1} \exp (\sqrt{-\lambda} x)+c_{2} \exp (-\sqrt{-\lambda} x),
$$

with generalized cofactor

$$
L_{\lambda}^{(-)}\left(\zeta^{(-)}, x\right)=\sqrt{-\lambda} \frac{c_{1} \exp (\sqrt{-\lambda} x)-c_{2} \exp (-\sqrt{-\lambda} x)}{c_{1} \exp (\sqrt{-\lambda} x)+c_{2} \exp (-\sqrt{-\lambda} x)} \in \mathbb{C}\left(\zeta^{(-)}, x, \exp (\sqrt{-\lambda} x)\right) .
$$

Generalized Darboux integrating factor $R_{\lambda}^{(-)}\left(\zeta^{(-)}, x\right)$ given by

$$
\frac{1}{\left(c_{1} \exp (\sqrt{-\lambda} x)+c_{2} \exp (-\sqrt{-\lambda} x)\right)^{2}\left(-\zeta^{(-)}+\sqrt{-\lambda} \frac{c_{1} \exp (\sqrt{-\lambda} x)-c_{2} \exp (-\sqrt{-\lambda} x)}{c_{1} \exp (\sqrt{-\lambda} x)+c_{2} \exp (-\sqrt{-\lambda} x)}\right)^{2}}
$$

and note that $R_{\lambda}^{(-)}\left(\zeta^{(-)}, x\right) \in \mathbb{C}\left(\zeta^{(-)}, x, \exp (\sqrt{-\lambda} x)\right)$.

Generalized Darboux first integral

$$
I_{\lambda}^{(-)}\left(\zeta^{(-)}, x\right)=\frac{-\zeta^{(-)}-\sqrt{-\lambda}}{-\zeta^{(-)}+\sqrt{-\lambda}} \exp (-2 \sqrt{-\lambda} x), \quad \lambda \neq 0
$$

and $I_{\lambda}^{(-)}\left(\zeta^{(-)}, x\right) \in \mathbb{C}\left(\zeta^{(-)}, x, \exp (\sqrt{-\lambda} x)\right)$. 
Applying the Darboux transformation DT for $\lambda \neq 0$ (and according to Proposition 5) we have

$$
\begin{aligned}
& \operatorname{DT}\left(\Psi_{\lambda}^{(-)}\right)=\Psi_{\lambda}^{(+)}=\Psi_{\lambda}^{(-) \prime}-\zeta_{0}^{(-)} \Psi_{\lambda}^{(-)} \\
&=\frac{c_{1}(\sqrt{-\lambda} x-1) \exp (\sqrt{-\lambda} x)}{x}-\frac{c_{2}(\sqrt{-\lambda} x+1) \exp (-\sqrt{-\lambda} x)}{x}, \\
& \zeta_{\lambda}^{(+)}=\left(\ln \left(\Psi_{\lambda}^{(+)}\right)\right)^{\prime}=-\frac{\lambda A x^{2}+\sqrt{-\lambda} B x-A}{x(\sqrt{-\lambda} B x-A)},
\end{aligned}
$$

with

$$
A=c_{1} \exp (\sqrt{-\lambda} x)+c_{2} \exp (-\sqrt{-\lambda} x), \quad B=c_{1} \exp (\sqrt{-\lambda} x)-c_{2} \exp (-\sqrt{-\lambda} x) .
$$

Additionally we have

$$
\begin{aligned}
& \zeta_{(\lambda, 1)}^{(+)}=\sqrt{-\lambda}+\left(\ln \left(\sqrt{-\lambda}-\frac{1}{x}\right)\right)^{\prime}=\sqrt{-\lambda}+\frac{1}{\sqrt{-\lambda} x^{2}-x}, \\
& \zeta_{(\lambda, 2)}^{(+)}=-\sqrt{-\lambda}+\left(\ln \left(-\sqrt{-\lambda}-\frac{1}{x}\right)\right)^{\prime}=-\sqrt{-\lambda}-\frac{1}{\sqrt{-\lambda} x^{2}+x} .
\end{aligned}
$$

We can see that for all $\lambda \in \Lambda$ the Picard-Vessiot extensions are given by $L_{0}=\widetilde{L}_{0}=\mathbb{C}(x), L_{\lambda}=$ $\widetilde{L}_{\lambda}=\mathbb{C}(x, \exp (\sqrt{-\lambda} x))$ for $\lambda \in \mathbb{C}^{*}$. In this way, we have that $\operatorname{DGal}\left(L_{0} / F\right)=\operatorname{DGal}\left(\widetilde{L}_{0} / F\right)=e$; for $\lambda \neq 0$, we have $\operatorname{DGal}\left(L_{\lambda} / F\right)=\operatorname{DGal}\left(\widetilde{L}_{\lambda} / F\right)=\mathbb{G}_{m}$, see $[1,3]$.

Now according to Proposition 5 we can compute $\mathrm{DT}\left(X_{\lambda}^{-}\right)=X_{\lambda}^{+}$and we obtain the rational vector field

$$
X_{\lambda}^{+}=\left(\frac{2}{x^{2}}-\lambda-\zeta^{(+)^{2}}\right) \frac{\partial}{\partial \zeta^{(+)}}+\frac{\partial}{\partial x}, \quad \forall \lambda \neq 0,
$$

and equivalently we can consider the polynomial vector field of degree four

$$
\bar{X}_{\lambda}^{+}=\left(2-\lambda x^{2}-x^{2} \zeta^{(+) 2}\right) \frac{\partial}{\partial \zeta^{(+)}}+x^{2} \frac{\partial}{\partial x}, \quad \forall \lambda \neq 0,
$$

and admits the following:

Invariant curve

$$
\begin{aligned}
\bar{f}_{\lambda}^{(+)}\left(\zeta^{(+)}, x\right) & =f_{\lambda}^{(+)}\left(\zeta^{(+)}, x\right)=-\zeta^{(+)}+\zeta_{\lambda}^{(-)}+\frac{\zeta_{\lambda}^{(-) \prime}+\frac{1}{x^{2}}}{\zeta_{\lambda}^{(-)}-\frac{1}{x}} \\
& =-\zeta^{(+)}-\frac{c_{1} A_{2} \exp (\sqrt{-\lambda} x)+c_{2} B_{2} \exp (-\sqrt{-\lambda} x)}{x\left(c_{1} A_{1} \exp (\sqrt{-\lambda} x)-c_{2} B_{1} \exp (-\sqrt{-\lambda} x)\right)},
\end{aligned}
$$

with

$$
A_{1}=\sqrt{-\lambda} x-1, \quad B_{1}=\sqrt{-\lambda} x+1, \quad A_{2}=\lambda x^{2}+A_{1}, \quad B_{2}=\lambda x^{2}-B_{1},
$$

and note that $f_{\lambda}^{(+)}\left(\zeta^{(+)}, x\right) \in \mathbb{C}\left(\zeta^{(+)}, x, \exp (\sqrt{-\lambda} x)\right)$ with generalized cofactor

$$
\begin{aligned}
\bar{K}_{\lambda}^{(+)}\left(\zeta^{(+)}, x\right) & =x^{2} K_{\lambda}^{(+)}\left(\zeta^{(+)}, x\right)=x^{2}\left(-\zeta^{(+)}-\zeta_{\lambda}^{(-)}-\frac{\zeta_{\lambda}^{(-) \prime}+\frac{1}{x^{2}}}{\zeta_{\lambda}^{(-)}-\frac{1}{x}}\right) \\
& =x^{2}\left(-\zeta^{(+)}+\frac{c_{1} A_{2} \exp (\sqrt{-\lambda} x)+c_{2} B_{2} \exp (-\sqrt{-\lambda} x)}{x\left(c_{1} A_{1} \exp (\sqrt{-\lambda} x)-c_{2} B_{1} \exp (-\sqrt{-\lambda} x)\right)}\right),
\end{aligned}
$$

and note that $K_{\lambda}^{(+)} \in \mathbb{C}\left(\zeta^{(+)}, x, \exp (\sqrt{-\lambda} x)\right)$. 
Generalized exponential factor

$$
\begin{aligned}
\bar{F}_{\lambda}^{(+)}\left(\zeta^{(+)}, x\right) & =F_{\lambda}^{(+)}\left(\zeta^{(+)}, x\right)=\left(\zeta_{\lambda}^{(-)}-\zeta_{0}^{(-)}\right) \exp \left(\int \zeta_{\lambda}^{(-)}\right) \\
& =\frac{c_{1} A_{1} \exp (\sqrt{-\lambda} x)+c_{2} B_{2} \exp (-\sqrt{-\lambda} x)}{x}
\end{aligned}
$$

with generalized cofactor

$$
\begin{aligned}
\bar{L}_{\lambda}^{(+)} & =x^{2} L_{\lambda}^{(+)}=x^{2} \zeta_{\lambda}^{(+)}=x^{2}\left(\zeta_{\lambda}^{(-)}+\frac{\zeta_{\lambda}^{(-) \prime}+\frac{1}{x^{2}}}{\zeta_{\lambda}^{(-)}-\frac{1}{x}}\right) \\
& =-x^{3} \frac{c_{1} A_{2} \exp (\sqrt{-\lambda} x)+c_{2} B_{2} \exp (-\sqrt{-\lambda})}{c_{1} A_{1} \exp (\sqrt{-\lambda} x)+c_{2} B_{2} \exp (-\sqrt{-\lambda} x)},
\end{aligned}
$$

and note that $L_{\lambda}^{(+)} \in \mathbb{C}\left(\zeta^{(+)}, x, \exp (\sqrt{-\lambda} x)\right)$.

Generalized Darboux integrating factor

$$
\bar{R}_{\lambda}^{(+)}\left(\zeta^{(+)}, x\right)=\frac{1}{x^{2}} R_{\lambda}^{(+)}\left(\zeta^{(+)}, x\right)=\frac{\left(c_{1} \exp (\sqrt{-\lambda} x)+c_{2} \exp (-\sqrt{-\lambda} x)\right)^{-2}}{x^{2}\left(\zeta_{\lambda}^{(-)}-\frac{1}{x}\right)^{2}\left(-\zeta^{(+)}+\zeta_{\lambda}^{(-)}+\frac{\zeta_{\lambda}^{(-) \prime}+\frac{1}{x^{2}}}{\zeta_{\lambda}^{(-)}-\frac{1}{x}}\right)^{2}},
$$

and $\bar{R}_{\lambda}^{(+)}\left(\zeta^{(+)}, x\right) \in \mathbb{C}\left(\zeta^{(+)}, x, \exp (\sqrt{-\lambda} x)\right)$.

Generalized Darboux first integral

$$
\bar{I}_{\lambda}^{(+)}\left(\zeta^{(+)}, x\right)=I_{\lambda}^{(+)}\left(\zeta^{(+)}, x\right)=\frac{-\zeta^{(+)}-\frac{1}{x B_{1}}-\sqrt{-\lambda}}{-\zeta^{(+)}+\frac{1}{x A_{1}}+\sqrt{-\lambda}}\left(\frac{B_{1}}{A_{1}}\right) \exp (-2 \sqrt{-\lambda} x),
$$

and $\bar{I}_{\lambda}^{(+)}\left(\zeta^{(+)}, x\right) \in \mathbb{C}\left(\zeta^{(+)}, x, \exp (\sqrt{-\lambda} x)\right)$.

We could consider $\zeta_{(\lambda, 1)}^{(-)}=-\sqrt{\lambda} \tan \sqrt{\lambda} x$, being $\Psi_{(\lambda, 1)}^{(-)}=\cos \sqrt{\lambda} x$ and $\Psi_{0}^{(-)}=x$. Thus we obtain the Schrödinger polynomial vector field

$$
X_{(\lambda, 1)}^{(-)}=\left(-\lambda-\zeta^{(-) 2}\right) \frac{\partial}{\partial \zeta^{(-)}}+\frac{\partial}{\partial x},
$$

of degree two. According to Lemma 1 for all $\lambda \in \Lambda \backslash\{0\}$, the vector field $X_{(\lambda, 1)}^{(-)}$admits the following:

Invariant curve

$$
f_{(\lambda, 1)}^{(-)}\left(\zeta^{(-)}, x\right)=-\zeta^{(-)}-\sqrt{\lambda} \tan (\sqrt{\lambda} x) \in \mathbb{C}\left(\zeta^{(-)}, x, \tan (\sqrt{\lambda} x)\right),
$$

with generalized cofactor

$$
K_{(\lambda, 1)}^{(-)}\left(\zeta^{(-)}, x\right)=-\zeta^{(-)}+\sqrt{\lambda} \tan (\sqrt{\lambda} x) \in \mathbb{C}\left(\zeta^{(-)}, x, \tan (\sqrt{\lambda} x)\right) .
$$

Generalized exponential factor

$$
F_{(\lambda, 1)}^{(-)}\left(\zeta^{(-)}, x\right)=\exp \left(-\sqrt{\lambda} \int \tan (\sqrt{\lambda} x) d x\right)=\frac{1}{\sqrt{1+\tan ^{2}(\sqrt{\lambda} x)}},
$$

with generalized cofactor $L_{(\lambda, 1)}^{(-)}\left(\zeta^{(-)}, x\right)=-\sqrt{\lambda} \tan (\sqrt{\lambda} x) \in \mathbb{C}\left(\zeta^{(-)}, x, \tan (\sqrt{\lambda} x)\right)$. 
Generalized Darboux integrating factor $($ for $\lambda \neq 0$ )

$$
R_{(\lambda, 1)}^{(-)}\left(\zeta^{(-)}, x\right)=\frac{1+\tan ^{2}(\sqrt{\lambda} x)}{\left(-\zeta^{(-)}-\sqrt{\lambda} \tan (\sqrt{\lambda} x)\right)^{2}} \in \mathbb{C}\left(\zeta^{(-)}, x, \tan (\sqrt{\lambda} x)\right) .
$$

Generalized Darboux first integral

$$
I_{(\lambda, 1)}^{(-)}\left(\zeta^{(-)}, x\right)=\frac{\zeta^{(-)} \tan (\sqrt{\lambda} x)-\sqrt{\lambda}}{\left.\left(\zeta^{(-)}+\sqrt{\lambda} \tan (\sqrt{(} \lambda) x\right)\right) \sqrt{\lambda}} \in \mathbb{C}\left(\zeta^{(-)}, x, \tan (\sqrt{\lambda} x)\right) .
$$

By Proposition 5, Darboux transformation of the vector field $X_{(\lambda, 1)}^{-}$is given by

$$
X_{(\lambda, 1)}^{+}=\left(\frac{2}{x^{2}}-\lambda-\zeta^{(+) 2}\right) \frac{\partial}{\partial \zeta^{(+)}}+\frac{\partial}{\partial x}
$$

and we can work with the polynomial vector field of degree four

$$
\bar{X}_{(\lambda, 1)}^{+}=\left(2-\lambda x^{2}-x^{2} \zeta^{(+) 2}\right) \frac{\partial}{\partial \zeta^{(+)}}+x^{2} \frac{\partial}{\partial x},
$$

that admits:

The invariant curve

$$
\bar{f}_{(\lambda, 1)}^{+}\left(\zeta^{(+)}, x\right)=-\zeta^{(+)}+\frac{-\sqrt{\lambda} x \tan (\sqrt{\lambda} x)+\lambda x^{2}-1}{x(\sqrt{\lambda} x \tan (\sqrt{\lambda} x)+1)},
$$

with generalized cofactor

$$
\bar{K}_{(\lambda, 1)}^{+}\left(\zeta^{(+)}, x\right)=x^{2}\left(-\zeta^{(+)}-\frac{-\sqrt{\lambda} x \tan (\sqrt{\lambda} x)+\lambda x^{2}-1}{x(\sqrt{\lambda} x \tan (\sqrt{\lambda} x)+1)}\right) .
$$

The generalized exponential factor

$$
\bar{F}_{(\lambda, 1)}^{+}\left(\zeta^{(+)}, x\right)=\frac{-\sqrt{\lambda} \tan (\sqrt{\lambda} x)-\frac{1}{x}}{\sqrt{1+\tan ^{2}(\sqrt{\lambda} x)}},
$$

with generalized cofactor

$$
\bar{L}_{(\lambda, 1)}^{+}\left(\zeta^{(+)}, x\right)=x \frac{-\sqrt{\lambda} x \tan (\sqrt{\lambda} x)+\lambda x^{2}-1}{\sqrt{\lambda} x \tan (\sqrt{\lambda} x)+1} .
$$

The integrating factor

$$
\bar{R}_{(\lambda, 1)}^{+}\left(\zeta^{(+)}, x\right)=\frac{1+\tan ^{2}(\sqrt{\lambda} x)}{x^{2}\left(-\zeta^{(+)}+\frac{\lambda x^{2}-A_{3}}{x A_{3}}\right)^{2}\left(-\frac{A_{3}}{x}\right)^{2}}
$$

with $A_{3}=\sqrt{\lambda} x \tan (\sqrt{\lambda} x)+1$.

Also we could consider the partial solution $\Psi_{(\lambda, 2)}^{(-)}=\sin (\sqrt{\lambda})$ and we could work in a similarly way as before. 
Remark 4. As in the same philosophy of the original Darboux transformation, we can iterate it to obtain families of new potentials. Starting with $V=0$, the following potentials can be obtained using Darboux iteration $\mathrm{DT}_{n}$ (see [4, 6, 22, 24, 25, 33, 42]):

$$
\begin{array}{rlrl}
\text { I) } & V_{n}=\frac{n(n-1) b^{2}}{(b x+c)^{2}}, & \text { II) } & V_{n}=\frac{m^{2} n(n-1)\left(b^{2}-a^{2}\right)}{(a \cosh (m x)+b \sinh (m x))^{2}}, \\
\text { III) } \quad V_{n}=\frac{-4 a b m^{2} n(n-1)}{(a \exp (m x)+b \exp (-m x))^{2}}, & \text { IV) } & V_{n}=\frac{m^{2} n(n-1)\left(b^{2}+a^{2}\right)}{(a \cos (m x)+b \sin (m x))^{2}} .
\end{array}
$$

In particular for the rational potential given in $I)$, see $[1,3]$, we have $F=F_{n}=\mathbb{C}(x)$ and for $\lambda_{n}=\lambda=0$, we have

$$
\Psi_{0}^{(n)}=\frac{c_{1}}{(b x+c)^{n}}+c_{2}(b x+c)^{n+1},
$$

so that

$$
\operatorname{DGal}\left(L_{0} / F\right)=\operatorname{DGal}\left(L_{0}^{(n)} / F\right)=e,
$$

whilst for $\lambda \neq 0$ and $\lambda_{n}=0$, the general solution $\Psi_{\lambda}^{(n)}$ is given by

$$
\Psi_{\lambda}^{(n)}(x)=c_{1} A_{n}(x, \lambda) C_{n}\left(\sin (\sqrt{\lambda} x)+c_{2} B_{n}(x, \lambda) D_{n}(\cos (\sqrt{\lambda} x))\right),
$$

where $A_{n}, B_{n}, C_{n}, D_{n} \in \mathbb{C}(x)$, so that

$$
\operatorname{DGal}\left(L_{\lambda} / F\right)=\operatorname{DGal}\left(L_{\lambda}^{(n)} / F\right)=\mathbb{G}_{m}
$$

Finally, considering $b=1, n=\ell+1, c=0$ and $x=r$ we arrive to the square well potential, which can be considered in a similar way.

Example 2 (3D Harmonic oscillator). We take $V_{-}=r^{2}+\frac{\ell(\ell+1)}{r^{2}}-(2 \ell+3)$, following [1, 3] we see that $\Lambda \cap \operatorname{Spec}(H)=4 \mathbb{N}$. For instance we consider $\lambda=4 n$, where $n \in \mathbb{N}$. Additionally, we have

$$
\begin{aligned}
\Psi_{0}^{(-)} & =r^{\ell+1} \exp \left(-\frac{r^{2}}{2}\right), & \zeta_{0}^{(-)} & =\frac{\ell+1}{r}-r \in \mathbb{C}(r), \quad \Psi_{n}^{(-)}=r^{\ell+1} P_{n} \exp \left(-\frac{r^{2}}{2}\right), \\
\zeta_{(n, 1)}^{(-)} & =\frac{\ell+1}{r}+\frac{P_{n}^{\prime}}{P_{n}}-r, & \zeta_{(n, 2)}^{(-)} & =\frac{\exp \left(r^{2}\right)}{r^{2 \ell+2} P_{n}^{2}} \int \frac{\exp \left(r^{2}\right)}{r^{2 \ell+2} P_{n}^{2}} d x+\frac{P_{n}^{\prime}}{P_{n}}-r+\frac{\ell+1}{r},
\end{aligned}
$$

where $P_{n}$ are polynomials of degree $n$ which are related to the generalized Laguerre polynomials, see [34]. According to Proposition 1 we see that DT is strong isogaloisian. Since,

$$
\mathrm{DT}\left(V_{-}\right)=V_{+}=r^{2}+\frac{(\ell+1)(\ell+2)}{r^{2}}-(2 \ell+1),
$$

we have that the potential $V_{-}$is also shape invariant.

Note that $T(r)=r^{4}+\ell(\ell+1)-(2 \ell+3) r^{2}$ and $N(r)=r^{2}$. Hence, system (15) can be written as

$$
X_{n}^{-}=\left(r^{4}+\ell(\ell+1)-(2 \ell+3) r^{2}-4 n r^{2}-r^{2} \zeta^{(-) 2}\right) \frac{\partial}{\partial \zeta^{(-)}}+r^{2} \frac{\partial}{\partial r} .
$$

According to Lemma 1 admits the invariant curve

$$
f_{n}^{(-)}\left(\zeta^{(-)}, r\right)=-\zeta^{(-)}+\frac{\ell+1}{r}+\frac{P_{n}^{\prime}}{P_{n}}-r \in \mathbb{C}\left(\zeta^{(-)}, r\right),
$$


with generalized cofactor

$$
K_{n}^{(-)}\left(\zeta^{(-)}, r\right)=-r^{2} \zeta^{(-)}-(\ell+1) r-\frac{P_{n}^{\prime}}{P_{n}} r+r^{2} \in \mathbb{C}\left(\zeta^{(-)}, r\right) .
$$

The generalized exponential factor

$$
F_{n}^{(-)}\left(\zeta^{(-)}, r\right)=r^{\ell+2} P_{n} \exp \left(\frac{-r^{2}}{2}\right) \in L^{2},
$$

with generalized cofactor

$$
L_{n}^{(-)}\left(\zeta^{(-)}, r\right)=r+(\ell+1) r+\frac{P_{n}^{\prime}}{P_{n}} r^{2}-r^{3} \in \mathbb{C}(r) .
$$

Moreover, $X_{\lambda}^{-}$admits the generalized Darboux integrating factor

$$
R_{n}^{(-)}\left(\zeta^{(-)}, r\right)=\frac{\exp \left(r^{2}\right)}{P_{n}^{2} r^{2(\ell+2)}\left(-\zeta^{(-)}+\frac{\ell+1}{r}+\frac{P_{n}^{\prime}}{P_{n}}-r\right)^{2}},
$$

where $R_{\lambda}^{(-)}\left(\zeta^{(-)}, r\right) \in \mathbb{C}\left(r, \exp \left(r^{2} / 2\right)\right)$ and the first integral

$$
I_{n}^{(-)}\left(\zeta^{(-)}, r\right)=\frac{\exp \left(r^{2}\right)}{r^{2 \ell+2} P_{n} P_{n}^{\prime}-r^{2 \ell+1} P_{n}^{2} A_{1}}+\int \frac{\exp \left(r^{2}\right)}{r^{2 \ell+1} P_{n}^{2}} d r \in F,
$$

with $A_{1}=r^{2}+\zeta^{(-)} r-\ell-1$. According to Proposition 5 using the Darboux transformation we obtain the rational vector field

$$
X_{n}^{+}=\left(r^{2}+\frac{(\ell+1)(\ell+2)}{r^{2}}-(2 \ell+1)-4 n-\zeta^{(+) 2}\right) \frac{\partial}{\partial \zeta^{(+)}}+\frac{\partial}{\partial r},
$$

or equivalently we can consider the polynomial vector field

$$
\bar{X}_{\lambda}^{+}=\left(r^{4}+(\ell+1)(\ell+2)-(2 \ell+1) r^{2}-4 n r^{2}-r^{2} \zeta^{(+) 2}\right) \frac{\partial}{\partial \zeta^{(+)}}+r^{2} \frac{\partial}{\partial r} .
$$

Moreover, we have

$$
\begin{aligned}
\zeta_{(n, 1)}^{(+)} & =\frac{P_{n}^{\prime \prime}}{P_{n}^{\prime}}-r+\frac{4 n+1}{r} \\
\zeta_{(n, 2)}^{(+)} & =r^{2 \ell+2} P_{n}\left(r P_{n}^{\prime \prime}-\left(r^{2}-\ell-1\right) P_{n}^{\prime}\right) \int \frac{\exp \left(r^{2}\right)}{r^{2 \ell+2} P_{n}^{2}}+\exp \left(r^{2}\right)\left(r^{2}-\ell-1\right) .
\end{aligned}
$$

The vector field $\bar{X}_{n}^{+}$admits the invariant curve

$$
\bar{f}_{n}^{(+)}\left(\zeta^{(+)}, r\right)=-\zeta^{(+)}+\frac{\ell+1}{r}+\frac{P_{n}^{\prime \prime}}{P_{n}^{\prime}}-r \in \mathbb{C}\left(\zeta^{(+)}, r\right),
$$

with generalized cofactor

$$
\bar{K}_{n}^{(+)}\left(\zeta^{(+)}, r\right)=-r^{2} \zeta^{(+)}-r(\ell+1)-\frac{P_{n}^{\prime \prime}}{P_{n}^{\prime}} r^{2}+r^{3} \in \mathbb{C}\left(\zeta^{(+)}, r\right) .
$$

The generalized exponential factor

$$
\bar{F}_{n}^{(+)}\left(\zeta^{(+)}, r\right)=r^{\ell+2} P_{n}^{\prime} \exp \left(-\frac{r^{2}}{2}\right) \in L^{2},
$$


with generalized cofactor

$$
\bar{L}_{n}^{(+)}\left(\zeta^{(+)}, r\right)=r+(\ell+1) r-\frac{P_{n}^{\prime \prime}}{P_{n}^{\prime}} r^{2}+r^{3} \in \mathbb{C}(r) .
$$

Additionally, the vector field $\bar{X}_{n}^{+}$admits the generalized Darboux integrating factor

$$
R_{n}^{(+)}\left(\zeta^{(+)}, r\right)=\frac{\exp \left(r^{2}\right)}{P_{n}^{\prime 2} r^{2(\ell+2)}\left(-\zeta^{(+)}+\frac{\ell+1}{r}+\frac{P_{n}^{\prime \prime}}{P_{n}^{\prime}}-r\right)^{2}} \in \mathbb{C}\left(r, \exp \left(r^{2} / 2\right)\right),
$$

and the first integral

$$
I_{n}^{(+)}\left(\zeta^{(+)}, r\right)=\frac{P_{n}^{\prime} P_{n} r^{2 \ell+2}\left(r P_{n}^{\prime \prime}-B_{2} P_{n}^{\prime}\right) I_{1}+A}{\left(r P_{n}^{\prime \prime}-B_{2} P_{n}^{\prime}\right)\left(\exp \left(r^{2}\right)+r^{2 \ell+2}\left(P_{n} P_{n}^{\prime}\right)^{2} I_{1}\right)},
$$

with

$$
\begin{aligned}
& I_{1}=\int \frac{\exp \left(r^{2}\right)}{r^{2 \ell+2} P_{n}^{2}} d r, \quad A=P_{n}^{\prime} \exp \left(r^{2}\right) B_{1} \exp \left(\int \Phi\right), \quad B_{1}=r^{2}-\zeta^{(+)} r-\ell-1, \\
& B_{2}=r^{2}+\zeta^{(+)} r-\ell-1, \quad \Phi=\frac{\exp \left(r^{2}\right)\left(\left(2 r^{2}-2 \ell-2\right) P_{n}^{\prime}-r P_{n}^{\prime \prime}\right)}{r P_{n}^{\prime}\left(\exp \left(r^{2}\right)+r^{2 \ell+2} P_{n} P_{n}^{\prime} \int \frac{\exp \left(r^{2}\right)}{r^{\ell \ell+2} P_{n}^{2}} d r\right)} .
\end{aligned}
$$

Note that $I_{n}^{( \pm)}\left(\zeta^{( \pm)}, r\right) \in F$, being $F$ an extension of $\mathbb{C}\left(r, \zeta^{( \pm)}\right)$adding the solutions of the Schrödinger equation $H^{ \pm} \Psi=\lambda \Psi$.

Example 3 (Coulomb). In this case we have $V_{-}=\frac{\ell(\ell+1)}{r^{2}}-\frac{2(\ell+1)}{r}+1$ and we can take

$$
\Psi_{0}^{(-)}=r^{\ell+1} \exp (-r), \quad \zeta_{0}^{(-)}=\frac{\ell+1}{r}-1 \in \mathbb{C}(r) .
$$

Note that due to Proposition 1 we have that DT is strong isogaloisian. Since

$$
\mathrm{DT}\left(V_{-}\right)=V_{+}=\frac{(\ell+1)(\ell+2)}{r^{2}}-\frac{2(\ell+1)}{r}+1
$$

we have that the potential $V_{-}$is shape invariant.

It is hold that

$$
\Lambda=\left\{1-\left(\frac{\ell+1}{\ell+1+n}\right)^{2}: n \in \mathbb{Z}_{+}\right\} \cup\left\{1-\left(\frac{\ell+1}{\ell-n}\right)^{2}: n \in \mathbb{Z}_{+}\right\},
$$

see $[1,3]$. In particular, for $\lambda=1-\left(\frac{\ell+1}{\ell+1+n}\right)^{2}, \lambda \neq 0$, we have

$$
\begin{aligned}
& \Psi_{n}^{(-)}=r^{\ell+1} P_{n} \exp \left(\frac{-(\ell+1) r}{\ell+1+n}\right), \quad \zeta_{(n, 1)}^{(-)}=\frac{\ell+1}{r}+\frac{P_{n}^{\prime}}{P_{n}}-\frac{\ell+1}{\ell+1+n} \in \mathbb{C}(r), \\
& \zeta_{(n, 2)}^{(+)}=\frac{\ell+1}{r}+\frac{P_{n}^{\prime}}{P_{n}}-\frac{\ell+1}{\ell+n+1}-1+\frac{\exp \left(\frac{4 r-2 n r}{\ell+n+1}\right)}{r^{2 \ell+2} P_{n}^{2} \int \frac{\exp \left(\frac{4 r-2 n r}{\ell+n+1}\right)}{r^{\ell+2} P_{n}^{2}}},
\end{aligned}
$$

where $P_{n}$ are polynomials of degree $n$, which are related to the generalized Laguerre polynomials $L_{n}^{(\ell)}$, for more details see [34]. 
According to Lemma 1 for $T(r)=\ell(\ell+1)-2(\ell+1) r+r^{2}$ and $N(r)=r^{2}$ the vector field (15) becomes

$$
X_{n}^{(-)}=\left(\ell(\ell+1)-2(\ell+1) r+\left(\frac{\ell+1}{\ell+1+n}\right)^{2} r^{2}-r^{2} \zeta^{(-) 2}\right) \frac{\partial}{\partial \zeta^{(-)}}+r^{2} \frac{\partial}{\partial r},
$$

and admits:

The invariant curve

$$
f_{n}^{(-)}\left(\zeta^{(-)}, r\right)=-\zeta^{(-)}+\frac{\ell+1}{r}+\frac{P_{n}^{\prime}}{P_{n}}-\frac{\ell+1}{\ell+1+n} \in \mathbb{C}\left(\zeta^{(-)}, r\right),
$$

with generalized cofactor

$$
K_{n}^{(-)}\left(\zeta^{(-)}, r\right)=-r^{2} \zeta^{(-)}-(\ell+1) r-\frac{P_{n}^{\prime}}{P_{n}} r^{2}+\frac{\ell+1}{\ell+1+n} r^{2} \in \mathbb{C}\left(\zeta^{(-)}, r\right) .
$$

The exponential factor

$$
F_{n}^{(-)}\left(\zeta^{(-)}, r\right)=r^{\ell+2} P_{n} \exp \left(\frac{-(\ell+1) r}{\ell+1+n}\right) \in L^{2},
$$

with generalized cofactor

$$
L_{n}^{(-)}\left(\zeta^{(-)}, r\right)=r+(\ell+1) r+\frac{P_{n}^{\prime}}{P_{n}} r^{2}-\frac{\ell+1}{\ell+1+n} r^{2} \in \mathbb{C}(r) .
$$

We note that the vector field $X_{n}^{-}$admits the generalized Darboux integrating factor

$$
R_{n}^{(-)}\left(\zeta^{(-)}, r\right)=\frac{\exp \left(\frac{2(\ell+1) r}{\ell+1+n}\right)}{P_{n}^{2} r^{2(\ell+2)}\left(-\zeta^{(-)}+\frac{\ell+1}{r}+\frac{P_{n}^{\prime}}{P_{n}}-\frac{\ell+1}{\ell+1+n}\right)^{2}} \in \mathbb{C}\left(\zeta^{(-)}, r, \exp \left(\frac{(\ell+1) r}{\ell+1+n}\right)\right) .
$$

The first integral

$$
I_{n}^{(-)}\left(\zeta^{(-)}, r\right)=\left(\frac{(\ell+n+1) r^{-2 \ell-1} \exp \left(\frac{4 r-2 n r}{\ell+n+1}\right)}{P_{n}\left((\ell+n+1) r P_{n}^{\prime}+(\ell+1)(\ell+n+1)-A I_{1}\right)}+1\right) \exp \left(\int \phi\right) \in F,
$$

with

$$
\begin{aligned}
& A=\ell r\left(\zeta^{(-)}+2\right)+n r\left(\zeta^{(-)}+1\right)+r\left(\zeta^{(-)}+2\right) P_{n}, \\
& I_{1}=\int \frac{\exp \left(\frac{4 r-2 n r}{\ell+n+1}\right)}{r^{2 \ell+2} P_{n}^{2}} d r, \quad \Phi=\frac{\exp \left(\frac{4 r-2 n r}{\ell+n+1}\right)}{r^{2 \ell+2} P_{n}^{2} \int \frac{\exp \left(\frac{4 r-2 n r}{\ell+n+1}\right)}{r^{2 \ell+2} P_{n}^{2}}} .
\end{aligned}
$$

Note that

$$
\begin{aligned}
\zeta_{(n, 1)}^{(+)}= & \frac{\ell+1}{r}-\frac{3 \ell+n+3}{\ell+n+1}+\frac{(\ell+n+1) P_{n}^{\prime \prime}}{\left((\ell+n+1) P_{n}^{\prime}-(\ell+1) P_{n}\right)} \\
& -\frac{(\ell+1)^{2} P_{n}}{(\ell+n+1)\left((\ell+n+1) P_{n}^{\prime}-(\ell+1) P_{n}\right)} .
\end{aligned}
$$


For suitability let assume $\mu=\ell+n+1$, thus we also have

$$
\zeta_{(n, 2)}^{(+)}=\frac{\exp \left(\frac{2 n r}{\mu}\right) r^{2 \ell+2} P_{n} \chi A_{1} P_{n}^{\prime \prime}+B_{1} P_{n}+\exp (4 r) \mu^{2}(r-\ell-1)}{r \mu\left(\exp \left(\frac{2 n r}{\mu}\right) r^{2 \ell+2} P_{n} \chi\left(\mu P_{n}^{\prime}-(\ell+1) P_{n}\right)+\mu \exp (4 r)\right)},
$$

with

$$
\begin{aligned}
& A_{1}=r \mu^{2} P_{n}^{\prime \prime}+\mu((\ell+1) \mu-r(3 \ell+n+3)), \quad B_{1}=(\ell+1)(r(2 \ell+n+2)-(\ell+1) \mu), \\
& \chi=\int \frac{\exp \left(\frac{4 r-2 n r}{\mu}\right)}{r^{2 \ell+2} P_{n}^{2}} d r .
\end{aligned}
$$

After the Darboux transformation DT the vector field $X_{n}^{-}$becomes

$$
X_{n}^{(+)}=\left(\frac{(\ell+1)(\ell+2)}{r^{2}}-\frac{2(\ell+1)}{r}+1-\lambda-\zeta^{(+) 2}\right) \frac{\partial}{\partial \zeta^{(+)}}+\frac{\partial}{\partial r} .
$$

The vector field $X_{n}^{+}$admits the invariant curve

$$
f_{n}^{(+)}\left(\zeta^{(+)}, r\right)=-\zeta^{(+)}+\frac{\ell+1}{r}+\frac{P_{n}^{\prime \prime}}{P_{n}^{\prime}}-\frac{\ell+1}{\ell+1+n} \in \mathbb{C}\left(\zeta^{(+)}, r\right),
$$

with generalized cofactor

$$
K_{n}^{(+)}\left(\zeta^{(+)}, r\right)=-\zeta^{(+)}-\frac{\ell+1}{r}-\frac{P_{n}^{\prime \prime}}{P_{n}^{\prime}}+\frac{\ell+1}{\ell+1+n} \in \mathbb{C}\left(\zeta^{(+)}, r\right) .
$$

The generalized exponential factor

$$
F_{n}^{(+)}\left(\zeta^{(+)}, r\right)=r^{\ell+1} P_{n}^{\prime} \exp \left(\frac{-(\ell+1) r}{\ell+1+n}\right) \in L^{2}
$$

with generalized cofactor

$$
L_{n}^{(+)}\left(\zeta^{(+)}, r\right)=\frac{\ell+1}{r}+\frac{P_{n}^{\prime \prime}}{P_{n}^{\prime}}-\frac{\ell+1}{\ell+1+n} \in \mathbb{C}(r) .
$$

Hence, the vector field $X_{n}^{+}$admits the generalized Darboux integrating factor

$$
R_{n}^{(+)}\left(\zeta^{(+)}, r\right)=\frac{\exp \left(\frac{2(\ell+1) r}{\ell+1+n}\right)}{P_{n}^{2} r^{2(\ell+1)}\left(-\zeta^{(+)}+\frac{\ell+1}{r}+\frac{P_{n}^{\prime \prime}}{P_{n}^{\prime}}-\frac{\ell+1}{\ell+1+n}\right)^{2}},
$$

and

$$
R_{n}^{(+)}\left(\zeta^{(+)}, r\right) \in \mathbb{C}\left(\zeta^{(+)}, r, \exp \left(\frac{(\ell+1) r}{\ell+1+n}\right)\right) .
$$

The first integral is given by relation

$$
I\left(\zeta^{(+)}, r\right)=\frac{-\zeta^{(+)}+\zeta_{(\lambda, 2)}^{(+)}}{-\zeta^{(+)}+\zeta_{(\lambda, 1)}^{(+)}} \exp \left(\int\left(\zeta_{(\lambda, 2)}^{(+)}-\zeta_{(\lambda, 1)}^{(+)}\right) d r\right) \in F .
$$

Note that $I_{n}^{( \pm)}\left(\zeta^{( \pm)}, r\right) \in F$, being $F$ an extension of $\mathbb{C}\left(r, \zeta^{( \pm)}\right)$adding the solutions of the Schrödinger equation $H^{ \pm} \Psi=\lambda \Psi$. 


\section{Final remarks}

This work is a first approach of applying Darboux transformation into polynomial vector fields that its associated foliation is of Riccati type. The strong isogaloisian property of the Darboux transformation guarantees that the transformed vector field is also of Riccati type. Additionally, we show that in the case of shape invariant potential is preserved the rational structure of the invariant objects such as the invariant curves, generalized cofactors, generalized Darboux integrating factors and Darboux first integrals. We remark that in this work we analyze the case of algebraically solvable potentials and in particular we consider potentials satisfying the shape invariance condition. A natural question arises: What happen in the case of quasi-algebraically solvable potentials? A more general study should be also done in the future for non rational potentials.

\section{Acknowledgements}

The authors are partial supported by the MICINN/FEDER grant number MTM2009-06973. The first author is also supported by Research Department of Universidad del Norte grant Agenda 2012. CP is additionally partially supported by the MICINN/FEDER grant MTM2008-03437 and by the Generalitat de Catalunya grant number 2009SGR859. The authors acknowledge to the anonymous referees by their useful comments and suggestions.

\section{References}

[1] Acosta-Humánez P.B., Galoisian approach to supersymmetric quantum mechanics. The integrability analysis of the Schrödinger equation by means of differential Galois theory, VDM Verlag, Dr Müller, Berlin, 2010.

[2] Acosta-Humánez P.B., Lázaro-Ochoa J.T., Morales-Ruiz J.J., Pantazi Ch., On the integrability of polynomial fields in the plane by means of Picard-Vessiot theory, arXiv:1012.4796.

[3] Acosta-Humánez P.B., Morales-Ruiz J.J., Weil J.A., Galoisian approach to integrability of Schrödinger equation, Rep. Math. Phys. 67 (2011), 305-374, arXiv:1008.3445.

[4] Berkovich L.M., Evlakhov S.A., The Euler-Imshenetskiı-Darboux transformation of second-order linear equations, Program. Comput. Software 32 (2006), 154-165.

[5] Blázquez-Sanz D., Pantazi Ch., A note on the Darboux theory of integrability of non autonomous polynomial differential systems, Preprint, 2011.

[6] Blecua P., Boya L.J., Segui A., New solvable quantum-mechanical potentials by iteration of the free $V=0$ potential, Nuovo Cimento B 118 (2003), 535-546, quant-ph/0311139.

[7] Carnicer M.M., The Poincaré problem in the nondicritical case, Ann. of Math. (2) 140 (1994), $289-294$.

[8] Cerveau D., Lins Neto A., Holomorphic foliations in $\mathbf{C P}(2)$ having an invariant algebraic curve, Ann. Inst. Fourier (Grenoble) 41 (1991), 883-903.

[9] Christopher C., Llibre J., Algebraic aspects of integrability for polynomial systems, Qual. Theory Dyn. Syst. 1 (1999), 71-95.

[10] Christopher C., Llibre J., Pantazi Ch., Walcher S., Inverse problems for invariant algebraic curves: explicit computations, Proc. Roy. Soc. Edinburgh Sect. A 139 (2009), 287-302.

[11] Christopher C., Llibre J., Pantazi Ch., Walcher S., Inverse problems for multiple invariant curves, Proc. Roy. Soc. Edinburgh Sect. A 137 (2007), 1197-1226.

[12] Christopher C., Llibre J., Pantazi Ch., Zhang X., Darboux integrability and invariant algebraic curves for planar polynomial systems, J. Phys. A: Math. Gen. 35 (2002), 2457-2476.

[13] Christopher C., Llibre J., Pereira J.V., Multiplicity of invariant algebraic curves in polynomial vector fields, Pacific J. Math. 229 (2007), 63-117.

[14] Cooper F., Khare A., Sukhatme U., Supersymmetry in quantum mechanics, World Scientific Publishing Co. Inc., River Edge, NJ, 2001. 
[15] Darboux G., Mémoire sur les équations différentielles algébriques du premier ordre et du premier degré, Bull. Sci. Math. (2) 2 (1878), 60-96, 123-144, 151-200.

[16] Darboux G., Sur une proposition relative aux équations linéaires, Comptes Rendus Acad. Sci. 94 (1882), 1456-1459.

[17] Darboux G., Théorie des Surfaces, II, Gauthier-Villars, Paris, 1889.

[18] García I.A., Giacomini H., Giné J., Generalized nonlinear superposition principles for polynomial planar vector fields, J. Lie Theory 15 (2005), 89-104.

[19] García I.A., Giné J., Generalized cofactors and nonlinear superposition principles, Appl. Math. Lett. 16 (2003), 1137-1141.

[20] Gendenshteïn L.E., Derivation of exact spectra of the Schrödinger equation by means of supersymmetry, JETP Lett. 38 (1983), 356-359.

[21] Giné J., Llibre J., A family of isochronous foci with Darboux first integral, Pacific J. Math. 218 (2005), 343-355.

[22] Ince E.L., Ordinary differential equations, Dover Publications, New York, 1944.

[23] Jouanolou J.P., Équations de Pfaff algébriques, Lecture Notes in Mathematics, Vol. 708, Springer, Berlin, 1979.

[24] Kalnins E.G., Kress J.M., Miller W., Families of classical subgroup separable superintegrable systems, J. Phys. A: Math. Theor. 43 (2010), 092001, 8 pages, arXiv:0912.3158.

[25] Kalnins E.G., Kress J.M., Miller W., Superintegrability and higher order integrals for quantum systems, J. Phys. A: Math. Theor. 43 (2010), 265205, 21 pages, arXiv:1002.2665.

[26] Kaplansky I., An introduction to differential algebra, Hermann, Paris, 1957.

[27] Kolchin E.R., Differential algebra and algebraic groups, Pure and Applied Mathematics, Vol. 54, Academic Press, New York, 1973.

[28] Kovacic J.J., An algorithm for solving second order linear homogeneous differential equations, J. Symbolic Comput. 2 (1986), 3-43.

[29] Llibre J., On the integrability of the differential systems in dimension two and of the polynomial differential systems in arbitrary dimension, J. Appl. Anal. Comput. 1 (2011), 33-52.

[30] Llibre J., Pantazi Ch., Darboux theory of integrability for a class of nonautonomous vector fields, J. Math. Phys. 50 (2009), 102705, 19 pages.

[31] Llibre J., Rodríguez G., Configurations of limit cycles and planar polynomial vector fields, J. Differential Equations 198 (2004), 374-380.

[32] Llibre J., Zhang X., Rational first integrals in the Darboux theory of integrability in $\mathbb{C}^{n}$, Bull. Sci. Math. 134 (2010), 189-195.

[33] Maciejewski A.J., Przybylska M., Yoshida H., Necessary conditions for classical super-integrability of a certain family of potentials in constant curvature spaces, J. Phys. A: Math. Theor. 43 (2010), 382001, 15 pages, arXiv:1004.3854.

[34] Nikiforov A.F., Uvarov V.B., Special functions of mathematical physics. A unified introduction with applications, Birkhäuser Verlag, Basel, 1988.

[35] Pantazi Ch., Inverse problems of the Darboux theory of integrability for planar polynomial differential systems, Ph.D. thesis, Universitat Autonoma de Barcelona, 2004.

[36] Prelle M.J., Singer M.F., Elementary first integrals of differential equations, Trans. Amer. Math. Soc. 279 (1983), 215-229.

[37] Ramis J.P., Martinet J., Théorie de Galois différentielle et resommation, in Computer Algebra and Differential Equations, Comput. Math. Appl., Academic Press, London, 1990, 117-214.

[38] Schlomiuk D., Algebraic particular integrals, integrability and the problem of the center, Trans. Amer. Math. Soc. 338 (1993), 799-841.

[39] Singer M.F., Liouvillian first integrals of differential equations, Trans. Amer. Math. Soc. 333 (1992), 673688.

[40] Spiridonov V., Universal superpositions of coherent states and self-similar potentials, Phys. Rev. A 52 (1995), 1909-1935, quant-ph/9601030. 
[41] Teschl G., Mathematical methods in quantum mechanics. With applications to Schrödinger operators, Graduate Studies in Mathematics, Vol. 99, American Mathematical Society, Providence, RI, 2009.

[42] Tremblay F., Turbiner A.V., Winternitz P., An infinite family of solvable and integrable quantum systems on a plane, J. Phys. A: Math. Theor. 42 (2009), 242001, 10 pages, arXiv:0904.0738.

[43] van der Put M., Singer M.F., Galois theory of linear differential equations, Grundlehren der Mathematischen Wissenschaften, Vol. 328, Springer-Verlag, Berlin, 2003.

[44] Weil J.A., Introduction to differential algebra and differential Galois theory, CIMPA-UNESCO Lectures, Hanoi, 2001.

[45] Witten E., Dynamical breaking of supersymmetry, Nuclear Phys. B 188 (1981), 513-554.

[46] Żołądek H., Polynomial Riccati equations with algebraic solutions, in Differential Galois Theory (Bȩdlewo, 2001), Banach Center Publ., Vol. 58, Polish Acad. Sci., Warsaw, 2002, 219-231. 